\title{
Biasing Amacrine Subtypes in the Atoh7 Lineage through Expression of Barhl2
}

\author{
Patricia R. Jusuf, ${ }^{1 \star}$ Shahad Albadri, ${ }^{2 \star}$ Alessio Paolini, ${ }^{2}$ Peter D. Currie, ${ }^{1}$ Francesco Argenton, ${ }^{3}$ Shin-ichi Higashijima, ${ }^{4}$ \\ William A. Harris, ${ }^{5}$ and Lucia Poggi ${ }^{2}$ \\ ${ }^{1}$ Australian Regenerative Medicine Institute, Monash University, Victoria 3800, Australia, ${ }^{2}$ Centre for Organismal Studies, University of Heidelberg, 69120 \\ Heidelberg, Germany, ${ }^{3}$ Department of Biology, University of Padua, 35131 Padua, Italy, ${ }^{4}$ National Institute for Physiological Sciences, Okazaki, Aichi, \\ 444-8585, Japan, and ${ }^{5}$ Department of Physiology, Development and Neuroscience, University of Cambridge, CB2 3DY Cambridge, United Kingdom
}

Within the developing vertebrate retina, particular subtypes of amacrine cells (ACs) tend to arise from progenitors expressing the basic helix-loop-helix (bHLH) transcription factor, Atoh7, which is necessary for the early generation of retinal ganglion cells (RGCs). All ACs require the postmitotic expression of the bHLH pancreas transcription factor Ptfla; however, Ptfla alone is not sufficient to give subtype identities. Here we use functional and in vivo time-lapse studies in the zebrafish retina to investigate on the developmental programs leading to ACs specification within the subsequent divisions of Atoh7-positive progenitors. We find evidences that the homeobox transcription factor Barhl2 is an AC subtype identity-biasing factor that turns on within Atoh7-positive descendants. In vivo lineage tracing reveals that particular modes of cell division tend to generate Barhl2-positive precursors from sisters of RGCs. Additionally, Atoh7 indirectly impacts these division modes to regulate the right number of barhl2-expressing cells. We finally find that Atoh7 itself influences the subtypes of Barhl2-dependent ACs. Together, the results from our study uncover lineage-related and molecular logic of subtype specification in the vertebrate retina, by showing that specific AC subtypes arise via a particular mode of cell division and a transcriptional network cascade involving the sequential expression of first atoh 7 followed by ptfla and then $b a r h l 2$.

\section{Introduction}

A major challenge in vertebrate neurobiology is to understand how the developmental program of a neural progenitor cell is regulated in vivo in the context of cell lineages and modes of cell division. Within the vertebrate retina, some types of neurons tend to be lineally related or descendants of common progenitor cells (Poggi et al., 2005b; Vitorino et al., 2009; Feng et al., 2010; Brzezinski et al., 2011; Jusuf et al., 2011). The bHLH transcription factor Atoh7 (also known as Ath5) is required for retinal ganglion cell (RGC) development (Brown et al., 2001; Kay et al., 2001; Vetter and Brown, 2001; Wang et al., 2001; Ghiasvand et al., 2011), and turns on just before mitosis that precedes their birth (Poggi et al., 2005b). One cell from this mitosis differentiates as a

Received April 30, 2012; revised Aug. 9, 2012; accepted Aug. 13, 2012.

Author contributions: P.R.J., S.A., and L.P. designed research; P.R.J., S.A., A.P., and L.P. performed research; P.D.C., F.A., S.-i.H., and W.A.H. contributed unpublished reagents/analytic tools; P.R.J., S.A., A.P., and L.P. analyzed data; P.R.J. and L.P. wrote the paper.

This work was supported by Deutsche Forschungsgemeinschaft Research Grant P0 1440/1-1 to L.P., Wellcome Trust Programme Grant ID RG49253 to W.A.H., and Australian National Health and Medical Research Council CJ Martin training fellowship ID 454798 to P.R.J. S.A. and A.P. are funded by Landesgraduiertenförderung (Funding program of the State of Baden-Württemberg). We thank J. L. Matéo Cerdán and D.S. Mosti for advice with statistical analyses; S. Schultz for help with Western blot experiments; and B. Wittbrodt, E. Leist, A. Saraceno, M. Majewski, B. Seiferling, T. Kellner, L. Schertel, C. Mueller, and Julian Cocks for fish maintenance and technical assistance. We are grateful to M. Carl, J. Wittbrodt, L. Centanin, G. Lupo, and M. Zigman for valuable discussion and comments on the manuscript. We also thank J. Wittbrodt for generous support.

*P.R.J. and S.A. contributed equally to this work.

Correspondence should be addressed to Lucia Poggi, Department of Molecular Developmental Biology and Physiology, Centre for Organismal Studies, Im Neuenheimer Feld 230, 69120 Heidelberg, Germany. E-mail: lucia.poggi@cos.uni-heidelberg.de.

DOI:10.1523/JNEUROSCI.2073-12.2012

Copyright $\odot 2012$ the authors $\quad 0270-6474 / 12 / 3213929-16 \$ 15.00 / 0$
RGC. However, many other cell types, including some subtypes of amacrine cells (ACs) also come from atoh7-expressing progenitors (Poggi et al., 2005b; Feng et al., 2010; Jusuf et al., 2011). The sisters of RGCs must therefore generate these other cell types.

The fates of all retinal neurons that primarily express the inhibitory neurotransmitters GABA or glycine (horizontal cells and ACs) require the expression of the pancreas transcription factor 1a (Ptfla) (Fujitani et al., 2006; Dullin et al., 2007; Nakhai et al., 2007; Jusuf et al., 2011). All ACs express Ptfla, but Ptfla alone is not sufficient to confer subtype specificity (Jusuf et al., 2011). However, precursors that express both atoh 7 and $p t f 1 a$ tend to differentiate into specific subtypes of ACs, thus suggesting that other key factors might regulate AC subtypes within this lineage (Jusuf et al., 2011).

Barhl homeobox transcription factors have been implicated in ACs diversity and RGC development downstream of Atoh7 (Poggi et al., 2004; Ding et al., 2009). Targeted disruption of barhl2 alters AC subtype composition and survival of RGCs (Ding et al., 2009). Nothing is known on the lineage-origin of barhl2-expressing cells, the networks in which Barhl2 specifies AC subtypes, or how it works in relation to genes that drive the same ( $p t f 1 a)$ or alternate fates (atoh7). In zebrafish, additional whole genome duplication has generated another barhl paralog (Reig et al., 2007; Schuhmacher et al., 2011). Barhl1.2 is specifically expressed in RGCs, while barhl2 is expressed in ACs (Schuhmacher et al., 2011). This led us to investigate the distinct role of Barhl2 as an AC subtype-biasing factor downstream of Atoh7. We found that barhl2-expressing precursors arise within the Atoh7-lineage. Barhl2 expression, however, does not depend on 
Atoh7, but on Ptfla, and is necessary and sufficient for biasing AC subtypes. Additionally, Atoh7 affects the identities of Barhl2dependent ACs. With time-lapse imaging (Poggi et al., 2005a,b) we traced the origins of Barhl2-positive cells. We found that these cells arise as one of the two postmitotic daughters of a dividing RGC's sister, i.e., Barhl2 ACs tend to be nieces of RGCs. Our study provides in vivo evidences that modes of cell division and lineage-restricted cell fate determination programs regulate the correct number of neuronal subtypes within particular progenitor pools.

\section{Materials and Methods}

Animals and ethics statements. Zebrafish breeding/raising followed standard protocols. Fish were maintained at $26.5^{\circ} \mathrm{C}$ and embryos raised at $28.5^{\circ} \mathrm{C}$ or $32^{\circ} \mathrm{C}$ and staged as described previously (Kimmel et al., 1995). Fish were housed in three facilities: Fish facility of our German laboratory (built in accordance to Tierschutzgesetz 111, Abs. 1, Nr. 1 and with European Union animal welfare guidelines); fish facility at the University of Cambridge, UK; and FishCore at Monash University, Australia. Each facility is under supervision of and in accordance with local animal welfare agencies. Zebrafish (Danio rerio) embryos of either sex were used exclusively before free-feeding stages. Embryos used for whole-mount imaging were treated with $0.0045 \%$ 1-phenyl-2-thiourea (Sigma) to delay pigment formation.

Fish lines. Seven transgenic lines expressing GFP, dsRed, gap43-GFP, or gap43-RFP under the control of different promoters were used in this study: $\operatorname{Tg}($ barhl2:GFP) line (Kinkhabwala et al., 2011); $\operatorname{Tg}$ (ptfla:GFP) line (Godinho et al., 2005) kindly provided by Steven D. Leach (Johns Hopkins Medical Institutions, Baltimore, $\mathrm{MD}) ; \operatorname{Tg}($ atoh7:gap43-GFP $), \operatorname{Tg}($ atoh7:GFP), $\operatorname{Tg}($ atoh7:gap43-RFP) and $\operatorname{Tg}$ (atoh7:gal4/pUAS:gap43-GFP) lines (Zolessi et al., 2006). For the $\operatorname{Tg}($ ptfla:dsRed $)$ line $\left(\operatorname{Tg}(-5.5 \text { ptfla:DsRed })_{\text {iac }}\right)$ we created a plasmid containing $5.5 \mathrm{~kb}$ of the $5^{\prime}$ region of the ptfla gene cloned upstream of DsRed2 in pT2AL200R150G vector (Kawakami, 2004). The plasmid was injected with Tol2 transposase mRNA and F1 progeny of different insertion lines was screened. The ia6 allele faithfully represents the endogenous expression of Ptfla mRNA and has a comparable expression pattern to that of the previously characterized $\operatorname{Tg}(p t f 1 a: G F P)$ with the dsRed showing only a slight delay in expression (data not shown). Double transgenic lines where generated via outcrossing.

Morpholino injection. Translation blocking morpholino oligonucleotides (MOs) obtained from Gene Tools, LLC were reconstituted as $1 \mathrm{~mm}$ stock solutions in water and injected into the yolk of 1-2 cell stage embryos. A MO targeted against a region $44 \mathrm{bp}$ upstream from the translational start site with sequence 5 ' -TTGCCCAGTAACAACAATCGCCTA C-3' was used to knockdown ptfla (10-12 ng/embryo) (Lin et al., 2004; Jusuf et al., 2011). A MO with sequence 5'TTCATGGCTCTTCAAAAA AGTCTCC-3' was used to knockdown atoh7 (Pittman et al., 2008). A barhl2 translation $\mathrm{MO}$ targeting $6 \mathrm{bp}$ upstream from the translational start site with the sequence $5^{\prime}$-AGAAAAGGATGAGCACTCAAGTCG $\mathrm{T}-3^{\prime}$ was designed and injected at $0.5 \mathrm{~mm} / \mathrm{embryo}$. Injections of standard control morpholino with sequence $5^{\prime}$-CCTCTTACCTCAGTTACAAT TTATA-3' up to 12 ng had no effect. The 5 bp Barhl 2 mismatch MO with sequence 5' -AGAATACGATCAGCACTGAACTCGT-3' is also comparable to uninjected (data not shown). Cell-autonomous role of Barhl2 was assessed using transplantation technique. Briefly, 10-20 cells were transplanted from blastula stage donors (cells labeled with H2A-GFP or $H 2 B-R F P$ RNA) into the animal poles of blastula stage host embryos. Integration and survival of transplanted cells was aided by injecting p53 MOs with sequence 5' -GCGCCATTGCTTTGCAAGAATTG-3' into donor embryos. Retinas injected with standard control MOs together with p53 MOs display normal expression of Barhl2 protein as shown by antibody staining (data not shown).

Overexpression plasmid cloning. The coding sequence of barhl2 was PCR amplified using 5' -ATGGAAGGATCCAGTGGGGCTAGT-3' and 5'-CCGAGCATGCGGTGTGCC-3' for which the reverse primer was tagged with the T2A sequence $5^{\prime}$-AGGGCCGGGATTCTCCTCCACGTC ACCGCATGTTAGAAGACTTCCTCTGCCCTC-3' (Kim et al., 2011). $H 2 B-R F P$ fused coding sequences were PCR amplified using $5^{\prime}$-ATGC
CAGAGCCAGCGAAGTCT-3' and 5'-GATGTACACGGCGCCGGT-3' primers for which the forward primer was tagged with the T2A sequence 5'-GAGGGCAGAGGAAGTCTTCTAACATGCGGTGACGTGGAGGAG AATCCCGGCCC-3'. A fusion PCR was performed to generate the barhl2$t 2 a-h 2 b-r f p$ product, which was cloned into a pUAS vector containing 16 cassettes of the Upstream Activation Sequence and recognition sequences for I-SceI meganuclease for efficient transgenesis.

Immunohistochemistry. Primary antibodies were diluted in blocking solution: rabbit anti-calretinin (Millipore Bioscience Research Reagents AB5054; 1:1000), rabbit anti-Sox2 (Millipore Bioscience Research Reagents AB5603, 1:200), mouse anti-parvalbumin (Millipore Bioscience Research Reagents MAB1572, 1:1000), rabbit anti-GABA (Sigma A2052, 1:500), rabbit anticalbindin (Calbiochem PC253L, 1:500), rabbit anti-serotonin (Sigma S5545, 1:50), mouse anti-tyrosine hydroxylase (Millipore MAB 318, 1:100), rabbit anti-Neuropeptide Y (Immunostar 22940, 1:500), rabbit anti-Barhl2 (Santa Cruz Biotechnology sc-68370, 1:50 for immunohistochemistry, 1:1000 for Western blot). Secondary antibodies were goat or donkey anti-mouse, antirabbit or anti-goat IgG conjugated to Alexa Fluor 488, 546, 594 or 647 fluorophores (1:1000-1:2000; Invitrogen).

For most antibodies, embryos were fixed in $4 \%$ paraformaldehyde (PFA) in $0.1 \mathrm{M}$ phosphate buffer (PB) overnight (maximum $2 \mathrm{~h}$ for ChAT immunohistochemistry) at $4^{\circ} \mathrm{C}$, rinsed, cryoprotected in $30 \%$ sucrose, embedded in OCT and cryosectioned at $14 \mu \mathrm{m}$ thickness. For GABA immunohistochemistry, embryos were fixed in $4 \%$ PFA/0.05\% glutaraldehyde, 5 mm EGTA, $5 \mathrm{~mm} \mathrm{MgSO}_{4}, 0.1 \%$ Triton X-100 in $0.1 \mathrm{M} \mathrm{PB}$ for $3 \mathrm{~h}$ at room temperature. All staining steps are performed at room temperature unless stated otherwise. For Sox 2 immunohistochemistry antigen retrieval was performed by immersing sections in $0.01 \mathrm{M}$ sodium citrate buffer, $\mathrm{pH} 6.0$ at $95^{\circ} \mathrm{C}$ for $10 \mathrm{~min}$ before blocking. All sections were incubated in blocking solution (10\% heat-inactivated goat serum, $1 \%$ bovine serum albumin, $0.2 \%$ Triton X-100 in PBS) for 30 min (sections) or $60 \mathrm{~min}$ (whole mounts). For staining with goat anti-ChAT antibody, sections were blocked in $10 \%$ donkey serum instead. Sections were incubated in primary antibodies overnight, secondary antibodies for $60 \mathrm{~min}$, and nuclei were counterstained with 4',6-diamidino-2-phenylindole (DAPI). Sections were mounted with Fluorosave (Calbiochem, Merck Chemicals Ltd.) or Mowiol.

Whole-mount single and fluorescent in situ hybridization. In situ mRNA hybridization was performed as described previously (Lin et al., 2004). The full-length cDNA barhl2 sequence was subcloned from a zebrafish IMAGE clone (IMAGE: 7452725; IRBOp991F0870D, Source BioScience UK Limited) in pME18S-FL3 into a pCS2+ vector to generate digoxigenin- and fluorescein-labeled riboprobes. For antisense probe, we linearized with NotI (Fermentas or New England Biolabs) and transcribed with Sp6 (mMessage mMachine Sp6, Ambion). For sense probe, we linearized with BamHI (Fermentas or New England Biology) and transcribed with T7 (mMessage mMachine T7, Ambion). Atoh7 probes were generated as described previously (Schuhmacher et al., 2011). Ptf1a probes were generated directly by RTPCR (one step RTPCR kit, Qiagen) using total mRNA extracted from zebrafish embryos of $50 \mathrm{~h}$ postfertilization (hpf) and 5'TTCGAGAGACCACTTGGACA-3' forward primer and T7 tailed 5' -CCAAGCTTCTAATACGACTCACTATAGGGAGAGG CTGAAACACAGATAGTCACAA-3' reverse primer. Single probe in situ hybridization was done as described previously (Thisse and Thisse, 2008) with minor modifications. Embryos underwent a stepwise dehydration series into $100 \%$ methanol and subsequent rehydration into $0.1 \%$ Tween in PBS. Permeabilization was achieved using age-dependent concentrations of proteinase $\mathrm{K}$ treatment at room temperature, followed by postfixation in 4\% PFA in PBS. After prehybridization, hybridization with digoxigenin-UTP-labeled probes (Roche Applied Science) was performed overnight at $65^{\circ} / 68^{\circ} \mathrm{C}$. Signal was detected with nitroblue tetrazolium chloride/5-bromo-4-chloro-3-indolyl-phosphate, toluidine salt (NBT/BCIP BM Purple, Roche Products Ltd.).

For double fluorescent whole mount in situ hybridization (FISH), standard digoxigenin- and fluorescein-labeled riboprobes were combined with Tyramide Signal Amplification, as described by Schuhmacher et al. (2011). Riboprobes were incubated for $30 \mathrm{~min}$ (barhl2), $40 \mathrm{~min}$ (atoh7), or $24 \mathrm{~h}$ ( $p t f 1 a)$. Embryos were kept in the dark for the following steps. Embryos were washed with TNT (0.1 M Tris, pH 7.5, $0.15 \mathrm{M} \mathrm{NaCl}$, 

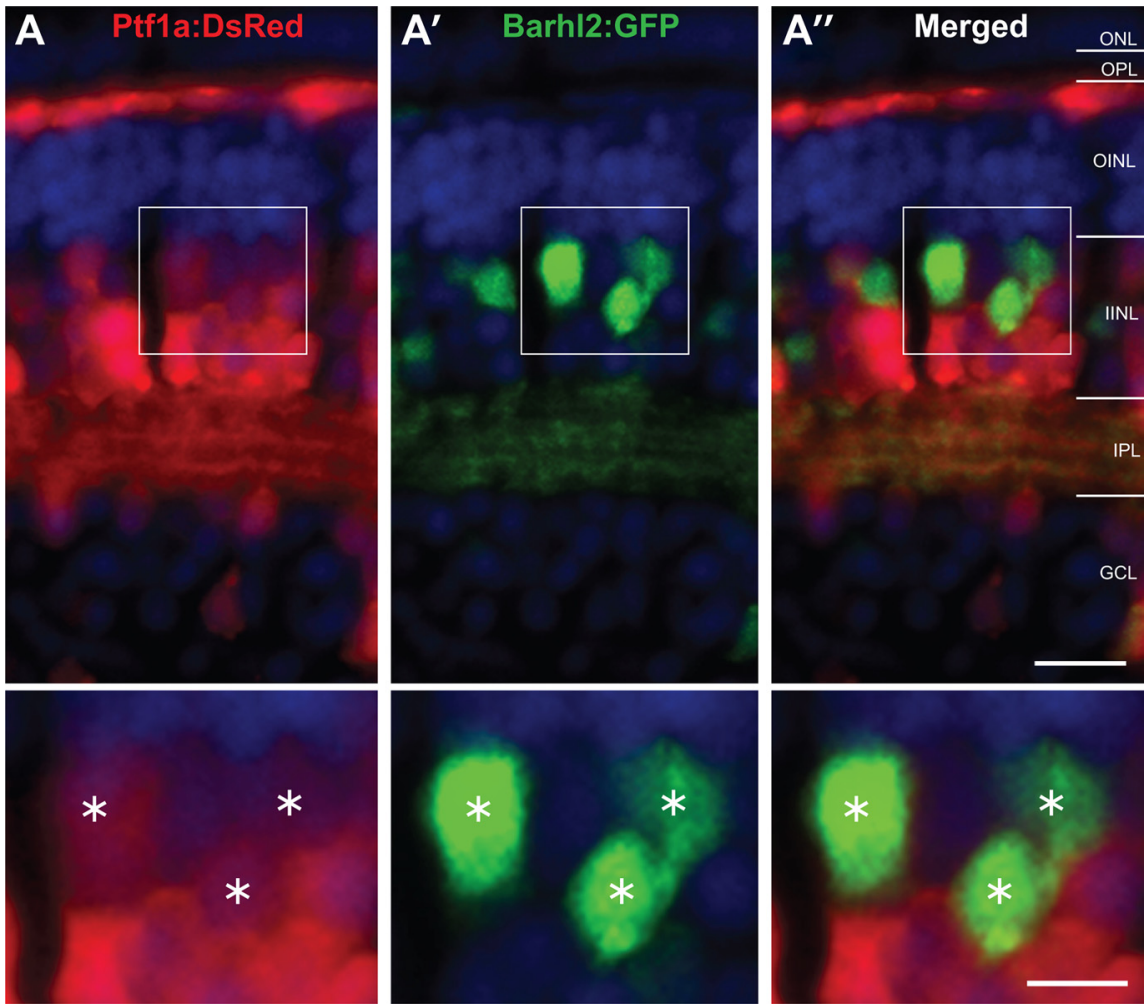

Figure 1. Barhl2:GFP labels a subpopulation of Ptfla:DsRed cells. Micrographs of retinal sections from Tg(ptf1a:dsRed/barhl2:GFP) showing red channel $(\boldsymbol{A})$, green channel $\left(\boldsymbol{A}^{\prime}\right)$ and merged channels $\left(\boldsymbol{A}^{\prime \prime}\right)$. Cell nuclei (in blue) are stained with DAPI. All of the Barhl2-positive cells are positive for varying degrees of Ptfla. In contrast, only some of the DsRed cells also express GFP, showing that a subpopulation of ptf1a-expressing cells expresses barh/2. Most of these double-labeled cells are found in the amacrine population in the INL. Results quantified from $n=58$ eyes, 5358 cells in central retina of $96 \mathrm{hpf}$ embryos. ONL, Outer nuclear layer; OPL, outer plexiform layer; OINL, outer half of the inner nuclear layer; IINL, inner half of the inner nuclear layer; IPL, inner plexiform layer; $G C L$, ganglion cell layer. Asterisks mark cells expressing both GFP and dsRed. Scale bars: (in $\boldsymbol{A}^{\prime \prime}$ ) $\boldsymbol{A}-\boldsymbol{A}^{\prime \prime}$, $20 \mu \mathrm{m}$; (in $\boldsymbol{A}^{\prime \prime}$ inset) $\boldsymbol{A}-\boldsymbol{A}^{\prime \prime}$ insets, $10 \mu \mathrm{m}$.

$0.1 \%$ Tween 20), incubated with $1 \% \mathrm{H}_{2} \mathrm{O}_{2}$ in TNT for 20 min, washed several times and blocked with TNB [ $2 \%$ DIG Block (Roche) in TNT] for $1 \mathrm{~h}$ followed by incubation with anti-digoxigenin-POD (peroxidase) Fab fragments (Roche, 1:50 in TNB). Signal was detected using fluoresceintyramide (FITC), cyanine3-tyramide (Cy3) or cyanine5-tyramide (Cy5) fluorophores (PerkinElmer). Embryos were incubated in DAPI in TNT overnight at $4^{\circ} \mathrm{C}$ and washed with TNT.

Imaging. NBT/BCIP-stained embryos were mounted in $87 \%$ glycerol and imaged with Leica DM5000B compound microscope at $10 \times$ or $20 \times$. Images were acquired with a Leica CD500 camera using Leica FireCam 1.7.1. FISH embryos were mounted on $100 \times 15 \mathrm{~mm}$ glass bottom Petri dishes using $1 \%$ low melting agarose and $z$-stacks taken at the Leica SP 5 confocal microscope.

Images of fixed and live embryos were acquired on a dissecting stereomicroscope equipped with epifluorescence (Leica MZ FLIII). Photomicrography of whole-mount eyes or sections was performed with a laser confocal system (Leica TCS-NT, Leica SpE or Leica Sp5 confocal laser scanning microscopes using a Leica $40 \times, 1.2 \mathrm{NA}$ or Leica $63 \times, 1.2 \mathrm{NA}$ water-immersion objectives) or Nikon fluorescence microscopes, equipped with cooled charge-coupled device (CCD) Hamamatsu Orca cameras and automated $z$-drive and fluorescence shutters.

At the confocal, excitation was achieved with following laser lines: 405 nm (DAPI), $488 \mathrm{~nm}$ argon (GFP, Alexa Fluor 488), $568 \mathrm{~nm}$ (RFP, DsRed, Alexa Fluor 546), $594 \mathrm{~nm}$ (Alexa Fluor 594) and $633 \mathrm{~nm}$ (Alexa Fluor 647). Images were taken through whole-mount fixed and live embryos as described previously (Poggi et al., 2005b). Sequential image acquisition was performed with emission detected at 500-550 nm (FITC), 650-700 $\mathrm{nm}$ (Cy3), 650-800 nm (Cy5) and 400-500 nm (DAPI) using individual descanned PMT detectors. Optical sections (40-60 $\mu \mathrm{m}$ for time-lapse and $<100 \mu \mathrm{m}$ for fixed embryos) of $1 \mu \mathrm{m}$ thickness were taken and Kalmann averaged 2 or 4 times. For time-lapse, images were taken every 5 or $10 \mathrm{~min}$ for $24-42 \mathrm{~h}$. Motorized XY stage was used to image multiple embryos. Laser power was minimized to avoid bleaching and phototoxicity.

Image processing and cell tracking. Image data were acquired using Leica Application Suite (LAS), Leica TCS NT or Leica LCS software, processed and analyzed using Volocity Analysis version 5.3 (Improvision). Brightness and contrast were adjusted with Adobe Photoshop CS3 and CS4.

Cell tracking was performed using the Volocity classification module (Improvision). Double-labeled Barhl2:GFP/Atoh7:gap43-RFP cells were randomly selected and Atoh7:gap43RFP-positive cells tracked backward in time.

Analysis. Numbers used for each analysis and age [generally 4 or 5 days postfertilization (dpf)] are indicated in the result section or figure legend. The majority of quantification was performed in the central retina in which the relatively older neurons express the proteins recognized by the antibodies. The region was defined by drawing a straight line through the center of the lens just central to the ciliary margin as described previously (Holt et al., 1988). The half covering the retina was subdivided into four segments and quantification performed in the central two. For transplanted cells of rare ACs, the whole retina was included. For markers with $<10$ cells per section, 5 sections were combined (i.e., serotonin, tyrosine hydroxylase and neuropeptide $\mathrm{Y}$ ).

Subtypes of barhl2-expressing AC subtypes were classified morphologically as described previously (Jusuf and Harris, 2009).

Statistical analysis. Statistical tests were performed using Prism software with $p<0.05$ used as criterion level. Comparison of morphologically characterized subtypes from barhl2expressing cells: Fisher's exact test. Tests used for atoh 7 knock down experiment: unpaired $t$ test with Welch's correction, Gaussian distribution, 2-tailed (GFP and GABA quantification); Fisher's exact test, 2 -sided and $\alpha<0.05$ (Serotonin and NY quantification). Tests used for barhl2 overexpression experiment with GABA: Mann-Whitney test, Gaussian approximation, 2-tailed. For barhl2 knockdown transplantation experiments, the binomial test was used, as the number of cells per image was too low to compare means.

\section{Results}

\section{Zebrafish barhl2 is expressed differentially in subtypes of inhibitory cells}

To investigate the possibility that the Barhl2 paralog in the zebrafish plays a specific role as an amacrine subtype specification factor within the Atoh7-lineage, we first assessed its gene expression with respect to the pancreas transcription factor $1 a$ (ptfla). Ptfla specifies all inhibitory (defined here as primarily expressing the inhibitory GABA or glycine neurotransmitters) neurons [ACs and horizontal cells (HCs)] and is excluded from excitatory (defined here as expressing the excitatory glutamate neurotransmitter) cells (Jusuf and Harris, 2009). We used the double transgenic line Tg(ptf1a:dsRed/barhl2:GFP) to assess whether cells expressing barhl2 also express ptfla. Quantification in $4 \mathrm{dpf} \operatorname{Tg}(p t f 1 a$ : dsRed/barhl2:GFP) transgenic embryos showed that Barhl2:GFP cells colabeled with Ptfla:DsRed signal (94.9 $\pm 0.5 \%$ SEM) primarily in ACs (Fig. 1). Additionally, the few Barhl2:GFP cells within the ganglion cell layer (GCL) coexpressed Ptf1a:DsRed, suggesting that these are displaced ACs. One striking observation was that, although all Barhl2:GFP cells are Ptfla-positive, only $\sim 58.5 \%$ ( $\pm 1.2 \%$ SEM) of Ptfla-expressing ACs are Barhl2:GFP- 
positive, suggesting that Barhl2 marks a subpopulation of Ptfla-derived ACs (Fig. 1). We found that some Ptfla:DsRed-positive HCs are also Barhl2:GFP-positive (Mo et al., 2004; Ding et al., 2009). These cells, however, are mainly located in the retinal periphery, and in these HCs, Barhl2:GFP expression was highly variable (data not shown). Thus, stable barhl2 paralog expression in the zebrafish retina is largely restricted to ACs.

As only $58.5 \%$ of Ptfla-positive ACs turn on Barhl2:GFP, we wondered whether barhl2 is expressed in specific subtypes. Previous studies implicated Barhl 2 in biasing specific AC identities (Yazulla and Studholme, 2001; Clemente et al., 2004), but a detailed characterization of subtypes expressing barhl2 and how this correlates with changes during loss- or gain-of-function studies are still missing. We therefore first performed immunohistochemical staining with nine antibodies in $5 \mathrm{dpf} \operatorname{Tg}$ (barhl2: GFP). We specifically chose a range of different markers. Serotonin, tyrosine hydroxylase, neuropeptide $\mathrm{Y}$, and choline acetyltransferase mark nonoverlapping individual subtypes based on colabeling or neurite morphology. Calbindin, parvalbumin and calretinin are calcium-binding markers that may label more than one subtype (e.g., parvalbumin labels 2 subtypes nonoverlapping with calretinin-labeled cells) (Yeo et al., 2009). Finally, we chose the more general marker GABA, which labels half of the zebrafish amacrine cells (Jusuf and Harris, 2009) and likely overlap with some of the subtype-specific markers (e.g., serotonin). We found that the vast majority of serotonin, calbindin and GABA-expressing AC types express Barhl2:GFP either strongly (serotonin) or at medium levels (calbindin, GABA; Fig. $2 A-C, J$ ), in line with GABAergic subpopulations overlapping with serotonin and/or calbindin-labeled subtypes. Calretinin, tyrosine hydroxylase or parvalbuminexpressing amacrine subtypes had more variability with approximately half of the labeled ACs expressing some Barhl2:GFP (Fig. 2D-F,J). Amacrine subtypes labeled by neuropeptide Y, Sox2 or choline acetyltransferase very rarely expressed Barhl2: GFP and never at high levels (Fig. 2G-I,J).

These observations are consistent with the hypothesis that Barhl2 may be involved in the differentiation of specific AC subtypes in the zebrafish retina and that differences in expression levels may be important in biasing subtype fates.

\section{Barhl2 functions downstream of Ptfla to bias amacrine subtypes identities}

As barhl2 is restricted to some AC subtypes, its expression might be turned on downstream of Ptfla only in certain AC precursor G) $A-I, 20 \mu \mathrm{m}$.
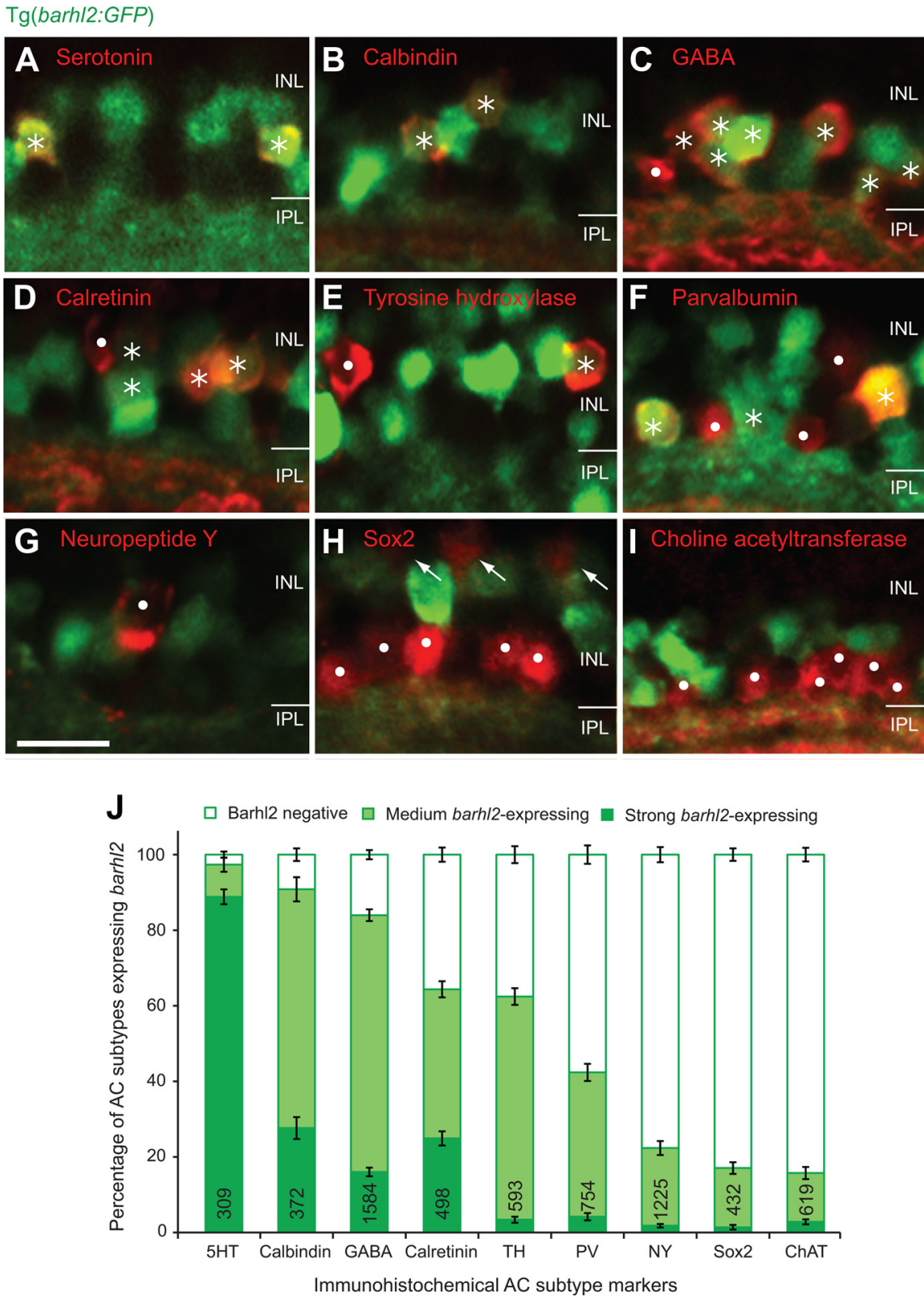

Figure 2. Amacrine markers reveal distinct subtypes expressing barh/2. Micrographs of $120 \mathrm{hpf} \mathrm{Tg}$ (barhl2:GFP) embryos immunohistochemically labeled with markers (red). $\boldsymbol{A}-\boldsymbol{C}$, Serotonin $(5 \mathrm{HT} ; \boldsymbol{A})$-, calbindin $(\boldsymbol{B})$-, and GABA ( $\boldsymbol{C}$-immunoreactive $A C$ types primarily colabel with Barhl2:GFP. D-F, Calretinin (D)-, tyrosine hydroxylase (TH; $\boldsymbol{E})$-, and parvalbumin (PV, $\boldsymbol{F}$ )-labeled populations show varying degrees and intensities of Barhl2:GFP colabeling. $\mathbf{G}-\boldsymbol{I}$, Neuropeptide Y (NY; $\boldsymbol{G})$, Sox2 $(\boldsymbol{H})$, and choline acetyltransferase (ChAT; $\boldsymbol{I}$ ) label ACs that do not colabel with Barhl2:GFP. Sox2 additionally labels Müller glia cells (arrows, $\boldsymbol{H}$ ). Asterisks indicate ACs (red) that colocalize with GFP; dots mark cells that do not express GFP.J, Quantification of the percentage of different markers colabeled with $\mathrm{Tg}($ barhl2:GFP), $n=21-220$ eyes, 309-1584 cells. ACs labeled by 5-HT, calbindin, and GABA primarily arise from barh/2-expressing cells. ACs labeled by NY, Sox2, and ChAT primarily come from Barhl2-negative or weakly barhl2expressing cells. ACs labeled with calretinin, TH, or PV include both, cells that do and do not arise from barhl2-expressing cells. Numbers in each bar indicate the number of labeled cells analyzed. Error bars indicate SEM. IPL, Inner plexiform layer. Scale bar: (in

populations to specify their identities. To first temporally locate barhl2 within the transcriptional cascade that leads to AC specification in vivo, we analyzed the dynamics of its expression at the cellular level with respect to ptfla. The first Barhl2:GFP signal starts at $35 \mathrm{hpf}$ and it faithfully recapitulates the endogenous barhl2 mRNA expression as revealed by double in situ hybridization with GFP mRNA (Fig. $3 A, B$ ) or GFP protein staining (Fig. $3 C, D) .3 \mathrm{D}$ time-lapse imaging starting from 30 to $32 \mathrm{hpf}$ show the first Barhl2:GFP signal at $35 \mathrm{hpf}$ (Movie 1). These Barhl2:GFP- 


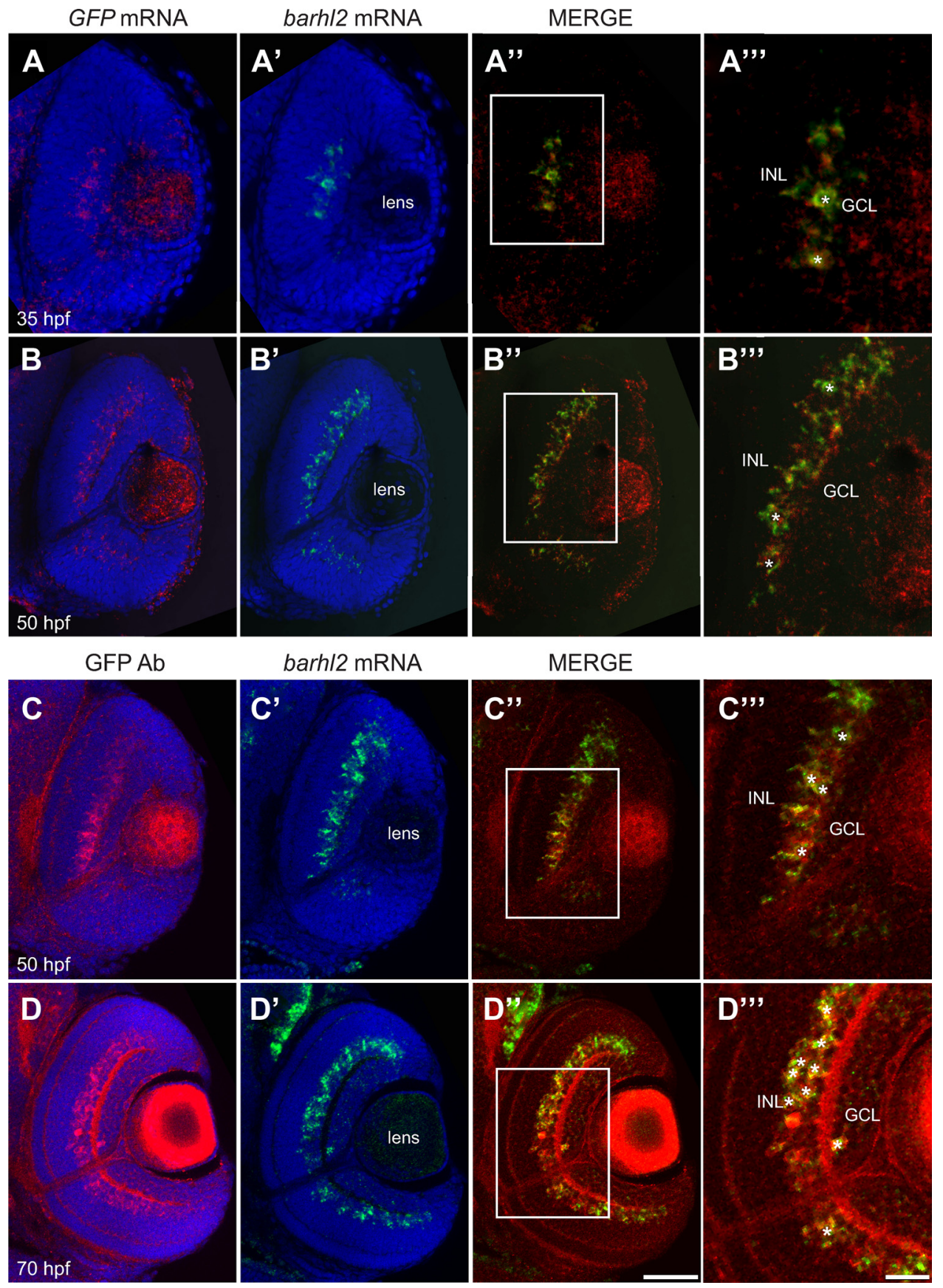

Figure 3. The $\operatorname{Tg}($ barh /2:GFP) transgenic line faithfully reflects the endogenous barh/2 expression in time and space. $A, B$, Whole-mount double fluorescent in situ hybridization against barh/2 mRNA and GFP mRNA on zebrafish retinas counterstained with DAPI (blue) at $35 \mathrm{hpf}\left(\boldsymbol{A}-\boldsymbol{A}^{\prime \prime \prime}\right)$ and $50 \mathrm{hpf}\left(\boldsymbol{B}-\boldsymbol{B}^{\prime \prime \prime}\right)$. C, $\boldsymbol{D}$, Whole-mount fluorescent in situ hybridization against barhl2 $\mathrm{mRNA}$ followed by immunohistochemical labeling against GFP at $50 \mathrm{hpf}\left(\boldsymbol{C}-\boldsymbol{C}^{\prime \prime \prime}\right)$ and $70 \mathrm{hpf}\left(\boldsymbol{D}-\boldsymbol{D}^{\prime \prime \prime}\right)$. Colocalization of barhl2 $\mathrm{mRNA}$ with barhl2 $\mathrm{mRNA}$ or Barhl2:GFP expression (asterisks) occurs in the INL at all ages and in few cells in the ganglion cell layer (GCL) at $70 \mathrm{hpf}$. Scale bars: (in $\left.\boldsymbol{D}^{\prime \prime}\right) \boldsymbol{A}-\boldsymbol{D}^{\prime \prime}, 50 \mu \mathrm{m}$; (in $\left.\boldsymbol{D}^{\prime \prime \prime}\right) \boldsymbol{A}^{\prime \prime \prime}-\boldsymbol{D}^{\prime \prime \prime}, 20 \mu \mathrm{m}$.

positive cells were never seen dividing, always being either in the process of or having finished migrating basally to the inner nuclear layer where ACs reside (Movie 1). Whole mount double fluorescent in situ hybridization shows coexpression of barhl2 and ptfla mRNAs in individual cells (Fig. 4A,B). Cells expressing ptfla only can be observed apically, suggesting that as cells migrate basally to the future AC layer, they first express ptfla. Consistently, our 3D in vivo time-lapse imaging shows that within individual developing neurons, Ptf1a:DsRed is turned on apically just after mitosis (Jusuf et al., 2011). In some of these cells Barhl2: GFP is turned on when they have reached the future AC layer (Fig. $4 C-C^{\prime \prime}$ ). We tested directly, if Barhl2 functions downstream of Ptfla, by injecting Ptfla translational $\mathrm{MO}$ or control $\mathrm{MO}$ into $\operatorname{Tg}($ barhl2:GFP) embryos. The Ptfla morphants show a drastic loss in the number of Barhl2:GFP cells (Fig. $4 D, E$ ) including ACs $(59.1 \pm 1.2$ SEM to $9.02 \pm 0.82$ SEM; $p<0.0001$; Fig. $4 F)$. Because Ptfla morphant cells remain within the retina and are respecified as excitatory cells (Jusuf et al., 2011), these results demonstrate that barhl2 turns on in inhibitory cell precursors downstream of Ptfla.

We assessed how the loss of Barhl2 affects the development of ptfla-expressing neurons. We used a MO, which effectively knocked down Barhl2 protein translation as shown by Western blot (50 hpf embryos) and antibody staining in hindbrain and 


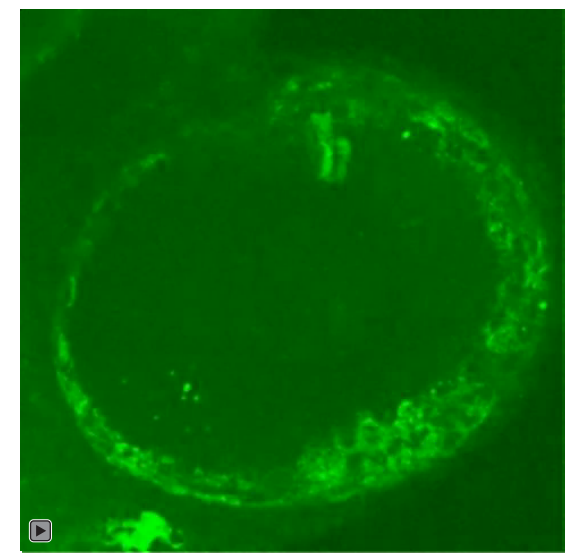

Movie 1. Time-lapse of developing retina revealing the timing and pattern of Barhl2:GFP onset in developing neurons from 35 to $50 \mathrm{hpf}$ ( $10 \mathrm{~min} /$ frame). In this transgenic zebrafish line, postmitotic barh/2-expressing cells first generate GFP as they migrate from the apical edge of the developing retina toward the inner retina. GFP-positive cells remain in the future amacrine layer.

retina (72 hpf embryos; Fig. 5). Standard control morpholinoinjected embryos were comparable to wild type (WT) (Fig. $5 F^{\prime}$ ), as were standard control $\mathrm{MO}+\mathrm{p} 53 \mathrm{MO}$ and $5 \mathrm{bp}$ mismatch $\mathrm{MO}$ (data not shown). As recent studies implicated Barhl2 in cell survival (Ding et al., 2009; Juraver-Geslin et al., 2011), we first assessed activated Caspase- 3 immunostaining in Barhl2 morphants, which revealed only a small nonsignificant increase in retinal apoptosis including in the AC layer [inner nuclear layer (INL), $p=0.3$; data not shown]. Thus, in the absence of Barhl2, the majority of inhibitory Ptfla:GFP cells remain in the appropriate layers. We next assessed the intrinsic effects of Barhl2 at single-cell level in a normal developing environment. For this we used the technical advantage of the zebrafish model to generate chimeras. Cells from embryos injected with Barhl2 MOs or control MOs were transplanted into wild-type host embryos (Fig. $6 A)$. We first transplanted cells from $\operatorname{Tg}(p t f 1 a$ :GFP) donors injected with $H 2 B-R F P$ mRNA (to label all donor cells). Overall, the Ptfla:GFP cells remained as inhibitory cells primarily in the inhibitory amacrine layer $(22.96 \%$ in WT to $21.2 \%$ in morphants, $p=0.13$, Fig. $6 B, C)$. The key question is whether the ACs generated in Barhl 2 morphants show changed subtype identity. Using transplantations from WT donors combined with immunohistochemistry, we indeed found that amacrine subtypes that usually express barhl2 were significantly lost in Barhl 2 morphant transplants (serotonin $43 \%, p=0.04$, calbindin 38\%, $p<0.0001$, GABA 68\%, $p<$ 0.0001 , Fig. $6 D-G, J)$. In contrast, some subtypes that usually do not express barhl2 were strikingly increased (neuropeptide Y 216\%, $p=$ 0.03 ), and others mildly increased (ChAT 125\%, $p=0.14$, Fig. $6 H-$ $J)$. No evidence was found for a role of Barhl2 in RGC differentiation or survival, as seen in other species (Mo et al., 2004; Poggi et al., 2005b; Ding et al., 2009). These observations demonstrate that the Barhl2 paralog in zebrafish is uniquely dedicated to the AC fates, in which it biases precursors toward generating specific some AC subtypes and away from others. These results also highlight a correlation between the expression level of barhl2 within distinct AC subtypes and its necessity during the development of each subtype.

\section{Barhl2 ACs are nieces of RGCs}

The results above demonstrate that Barhl2 acts downstream of Ptfla to bias specific subtype identities within a subset of postmi- totic AC precursors. How does this cell population and transcriptional cascade relate to the Atoh7-lineage in vivo? Approximately $2 / 3$ of the AC population arises from atoh7-expressing progenitors (Jusuf et al., 2011). Since we found that barhl2-expressing neurons comprise $58.5 \%$ of $p t f 1 a$-expressing ACs, we wondered whether barhl2-expressing ACs come from atoh7-expressing progenitors. Previous expression analysis on barhl2 and atoh7 did not provide any evidence for mRNA colocalization, e.g., RGCs express atoh7 and not barhl2 and ACs that express barhl2 do not express atoh7 (Schuhmacher et al., 2011). This kind of approach, however, cannot rule out possible lineage-relationships between cells expressing these two factors. In contrast, some of the fluorescent proteins display long perdurance compared with the native mRNAs under whose promoters they are driven. Thus, in the $\operatorname{Tg}$ (barhl2:GFP/atoh7:gap43-RFP) double transgenic line in which the RFP is long-lived compared with atoh 7 mRNA, we can visualize the respective onset of Barhl2:GFP and Atoh7:gap43$\mathrm{RFP}$ in individual cells using in vivo $3 \mathrm{D}$ time-lapse imaging. We started imaging at $\sim 35 \mathrm{hpf}$, when retinal neurons first express Barhl2:GFP, and asked whether any Barhl2:GFP-positive neurons arise from atoh7-expressing progenitors within the first $6 \mathrm{~h}$ of each time-lapse movie (Fig. 7A). Strikingly, we found that almost all of the Barhl2:GFP-expressing cells in this time-window also were Atoh7:gap43-RFP-positives (94.3 $\pm 2.8 \%$ SEM), suggesting that Barhl2-dependent amacrine subtypes indeed arise from the Atoh7-lineage. We compared the distribution of subtypes that express barhl2 with our previously identified subtype biases within the Atoh7-lineage (Jusuf et al., 2011). We thus performed morphological characterization of individual Barhl2: GFP expressing cells $(n=28$, Fig. $7 B-D)$ and compared the frequency of subtypes in three categories: We found that Barhl2: GFP subtypes of ACs are underrepresented or overrepresented at similar frequency as are Atoh7+/Ptfla +-expressing cells when compared with all $(\mathrm{Ptfla}+)$ subtype frequencies. Thus, our Barhl2 + sample was statistically significantly different from WT $(p=0.032)$, but comparable to the Atoh7 $+/ \mathrm{Ptfla}+$ population $(p=0.55)$, which our time-lapse analysis showed to be the origin of barhl2-expressing cells.

If Barhl2-dependent ACs derive from the Atoh7-lineage, then time-lapse imaging should allow us to understand the lineage relationship between barhl2-expressing amacrine subtypes and RGCs. In our previous 3D time-lapse study we traced individual dividing Atoh7:GFP-expressing progenitors long enough to show that these cells often divided asymmetrically to produce one daughter cell that became a RGC and another daughter which often migrated back toward the apical surface (Poggi et al., 2005b). The time-lapse limitations and lack of appropriate fluorescent reporters did not allow us to follow the fate of the nonRGC daughter cell for more than a few hours, during which time they did not divide again (Poggi et al., 2005b). In the Tg(barhl2: GFP/atoh7:gap43-RFP) double transgenic line, however, it is possible to trace the cellular origin of Barhl2:GFP/Atoh7:gap43RFP-positive cells. Seven retinas were imaged for a minimum of $20 \mathrm{~h}$ starting from $28 \mathrm{hpf}$. We traced the lineage of 20 individual Barhl2:GFP/Atoh7:gap43-RFP-positive cells. Strikingly, in 19 of these 20 cases, the Barhl2:GFP-positive cell arose from a cell division of an Atoh7:gap43-RFP-positive progenitor, which was identified as the sister of an RGC (Movie 2). In all these 19 cases, the Atoh7:gap43-RFP progenitor generated one Barhl2-positive and one Barhl2-negative daughter cell whose identity remains unknown (Movie 2). In one case of the 20, we observed that both daughters became Barhl2:GFP-positive. In 14 cases, we were able 


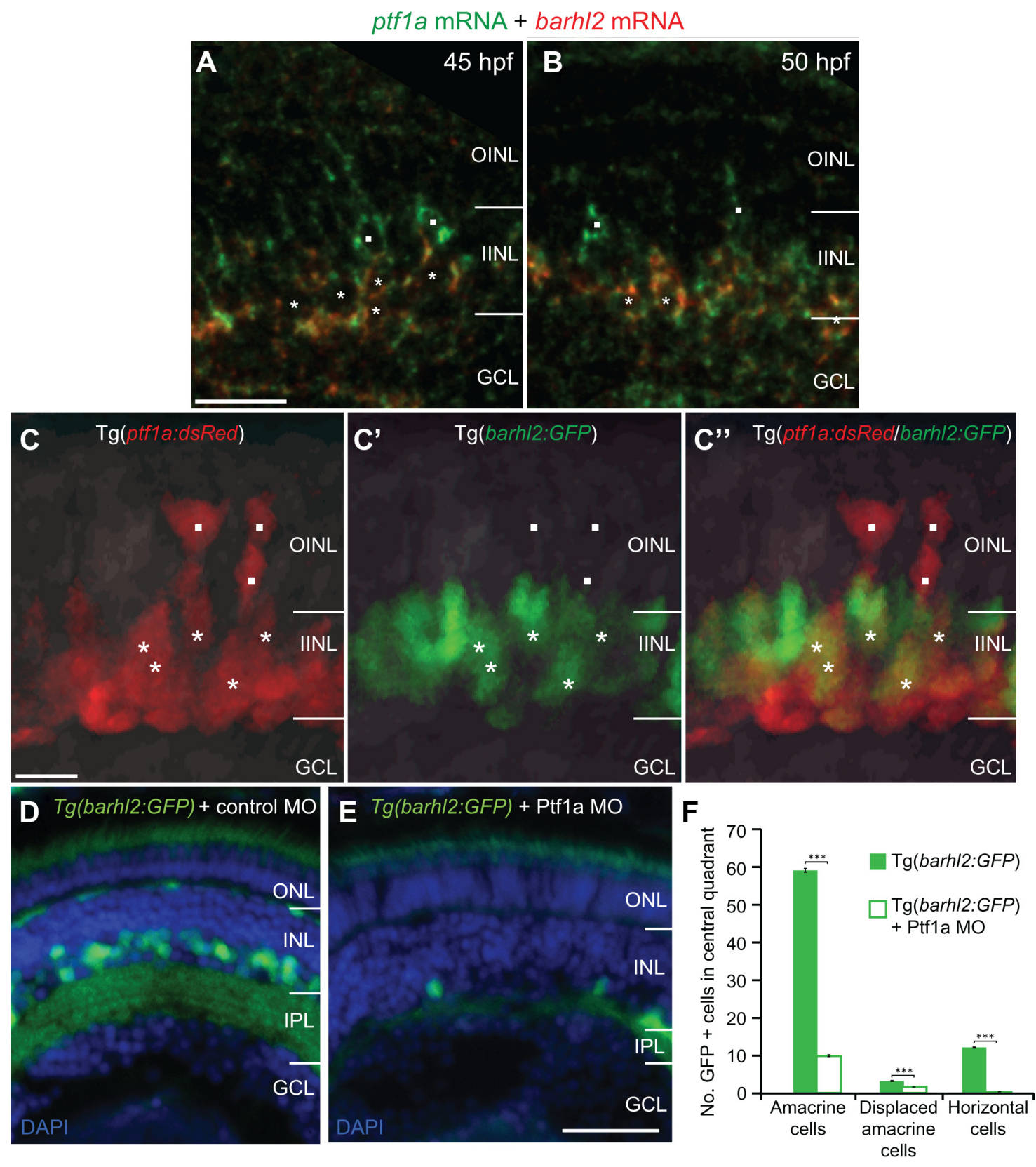

Figure 4. Ptf1a/barh/2 are sequentially expressed within individual cells, with Ptf1a being necessary for Barhl2:GFP expression. $A$, B, Double fluorescent in situ hybridization of barhl2 and ptf1a mRNAs. Ptfla (green) is expressed in cells apically (squares) and in cells that have migrated basally where they also express barhl2 (red, asterisks) at $45 \mathrm{hpf}(\boldsymbol{A})$ and $50 \mathrm{hpf}(\boldsymbol{B}) . \boldsymbol{C}-\boldsymbol{C}^{\prime}, \mathbf{M i c r o g r a p h s}$ from in vivo time-lapse of double transgenic Tg(ptf1a:dsRed/barh/2:GFP) embryos at $35 \mathrm{hpf}$ show a similar pattern with apical cells expressing Ptf1a:DsRed alone (squares) and more basal cells coexpressing Barhl2:GFP (asterisks). D, E, Micrographs of $120 \mathrm{hpf} \mathrm{Tg(barh/2:GFP)} \mathrm{injected} \mathrm{with} \mathrm{standard} \mathrm{morpholino} \mathrm{(D)} \mathrm{or} \mathrm{Ptf1a} \mathrm{morpholino} \mathrm{(E).} \mathrm{Barhl2:GFP} \mathrm{expression} \mathrm{is} \mathrm{drastically} \mathrm{reduced} \mathrm{in} \mathrm{the}$ Ptf1a morphants. F, Quantification of GFP-labeled cells shows a significant loss of cells in Ptf1a MO in all cell types that usually express Barhl2:GFP, i.e., ACs (inner half of INL), displaced ACs (outermost layer of $\mathrm{GCL}$ ) and horizontal cells (outermost layer of INL). WT $n=51$ eyes, Ptfla morphant $n=41$ eyes. ONL, Outer nuclear layer; IPL, inner plexiform layer; $\mathrm{GCL}$, ganglion cell layer. ${ }^{* * *} p<0.001$; error bars indicate SEM. Scale bars: (in $\boldsymbol{A}) \boldsymbol{A}, \boldsymbol{B}, 20 \mu \mathrm{m}$; (in $\boldsymbol{C}) \boldsymbol{C}-\boldsymbol{C}^{\prime \prime}, 10 \mu \mathrm{m}$; (in $\left.\boldsymbol{E}\right) \boldsymbol{D}, \boldsymbol{E}, 50 \mu \mathrm{m}$.

to reconstruct the lineage of the Barhl2-positive AC from the very onset of Atoh7:gap43-RFP expression. In all of these cases, the lineage started with the asymmetrical division of an Atoh7positive progenitor, one daughter of which turned up Atoh7: gap43-RFP expression and became a RGC, while the other daughter divided again (see example in Fig. $7 E$ ). The result of this next division was one Barhl2:GFP-positive cell and one Barhl2:GFP-negative cell (Fig. 7E; Movie 2). Together, these observations suggest that during RGC genesis in vivo, Barhl2positive ACs arise as nieces of RGCs, mainly through asymmetric fate outcome of atoh7-expressing progenitors division (Fig. $7 F)$.

\section{Barhl2 acts within the Atoh7-lineage to specify subtypes} of ACs

Barhl2 is expressed in cells that derive from Atoh7-progenitors, and biases AC identities toward the same subtype identities that arise in the Atoh7-lineage. Therefore, it is important to determine whether Barhl2 by itself is capable in biasing amacrine subtypes in vivo. We investigated this by expressing barhl2 within atoh7expressing progenitors such that all rather than a subset of these cells would turn on Barhl2. DNA for the H2B-RFP reporter was injected into 1-cell stage embryos in which either $p U A S: H 2 B-R F P$ (in control retinas) or from $p U A S$ :barhl2-T2A-H2B-RFP (barhl2 misexpressing retinas) was expressed under the control of atoh7 
A

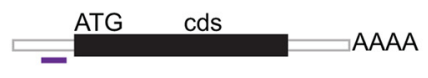

BARHL2 MO

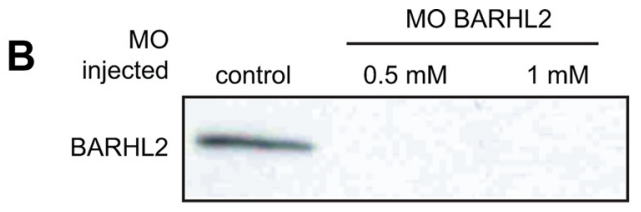

UBC9
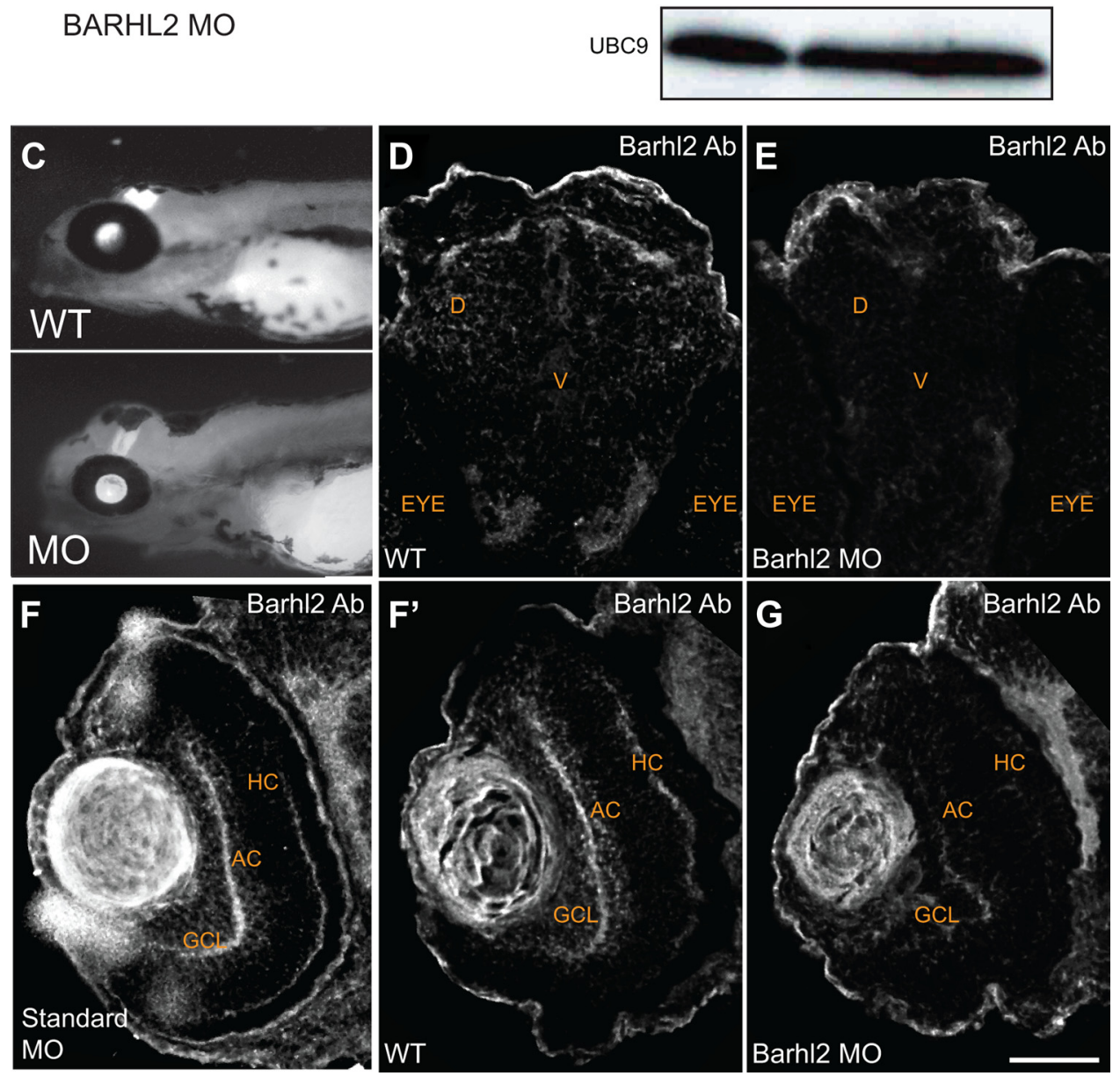

Figure 5. Zebrafish Barhl2 morpholino causes efficient barhl2 knockdown. $A, A$ translational blocking $M 0$ was designed against 6 base pairs upstream the translational start site of zebrafish barh/2 mRNA. The morpholino efficiency was tested using anti-Barhl2 antibody. B, Western Blotting of wild-type (control) and morphant (0.5 and 1 mm Barhl2 M0) 50-hpf-old embryos heads. C, Micrographs of control and Barhl2 morphant embryos. Morphants look relatively normal, showing only mild general defects in their heads. $\boldsymbol{D}-\boldsymbol{G}, \mathbf{I m m u n o h i s t o c h e m i c a l ~ l a b e l i n g ~ o f ~ b r a i n ~}(\boldsymbol{D}, \boldsymbol{E})$ and retinal $\left(\boldsymbol{F}, \boldsymbol{F}^{\prime}, \boldsymbol{G}\right)$ sections at $50 \mathrm{hpf}$. Control embryos $(\boldsymbol{D}, \boldsymbol{F})$ and standard $\mathrm{M} 0$-injected embryos $\left(\boldsymbol{F}^{\prime}\right)$ show Barhl2 protein in the diencephalon region $\mathrm{D}$ around the ventricular zone $V$ of the brain $(\boldsymbol{D})$ and in the retina $\left(\boldsymbol{F}, \boldsymbol{F}^{\prime}\right)$, in ACs and HCs and in cells located in the ganglion cell layer $\left(\mathrm{GCL} ; \boldsymbol{F}, \boldsymbol{F}^{\prime}\right)$. Barhl2 morphant embryo brain and retina do not stain for Barhl2 protein. Scale bar: (in G) $D-G, 50 \mu \mathrm{m}$.

promoter in the $\operatorname{Tg}$ (atoh7:gal4/pUAS:gap43-GFP) line. The use of Barhl2-T2A-H2B-RFP in frame fusion allows visualization of barhl2-expressing cells in vivo, and allows simultaneous assessment of their final fate (Kim et al., 2011). In vivo time-lapse analysis confirmed that the expression of barhl2 (H2B-RFPpositive cells) indeed occurred prematurely, in dividing Atoh7positive progenitors (Movie 3). Cell fates of H2B-RFP-labeled cells were quantified at $4 \mathrm{dpf}$ (Fig. 8). We found no significant changes in the proportion of H2B-RFP-positive ACs in the INL ( $21.02 \pm 4.08 \%$ SEM control to $26.9 \pm 4.15 \%$ SEM misexpression, $p=0.3255)$ or HCs $(20.26 \pm 5.11 \%$ SEM control to $19.26 \pm$ $4.0 \%$ SEM misexpression, $p=0.5157$ ), nor bipolar cells in the outer half of the INL $(8.2 \pm 5.02 \%$ SEM control to $8.9 \pm 3.97 \%$ SEM misexpression, $p=0.1404$ ). However, barhl2 misexpression leads to a significant increase of H2B-RFP-positive cells in the GCL (17.68 $\pm 5.36 \%$ SEM control to $34.82 \pm 5.8 \%$ SEM misexpression, $p=0.0017$ ) and a significant loss of H2B-RFP-positive cells among photoreceptor (the outer nuclear layer $(\mathrm{ONL})$ ) cells
(32.84 $\pm 5.76 \%$ SEM control to $10.12 \pm 5.4 \%$ SEM misexpression, $p=0.0105)$. To analyze this effect with respect to AC subtypes, we used antibodies for specific AC populations. Within barhl2-misexpressing H2B-RFP-positive cells we found a significant increase in the proportion of GABAergic cells in the INL $(15.93 \pm 4.86 \%$ SEM control to $22.2 \pm 4.64 \%$ SEM misexpression, $p=0.0044)$ and GCL $(7.5 \pm 4 \%$ SEM control to $28.32 \pm$ $6.05 \%$ SEM misexpression, $p<0.0001$ ), which was particularly evident in the GCL (Fig. $8 D$ ). Therefore, the increase in GABA + cells appears to be at the expense of early made ganglion cells (GABA - in the GCL) and photoreceptors. Notably, neither in the control nor in the misexpression condition were H2B-RFP-positive cells ever ChAT-positive (data not shown), a subtype that rarely expresses barhl2. These results suggest that when barhl2 is prematurely expressed in the atoh7expressing progenitors population, it is itself able to induce within these cells some aspects of Barhl2-dependent AC subtype identities. 

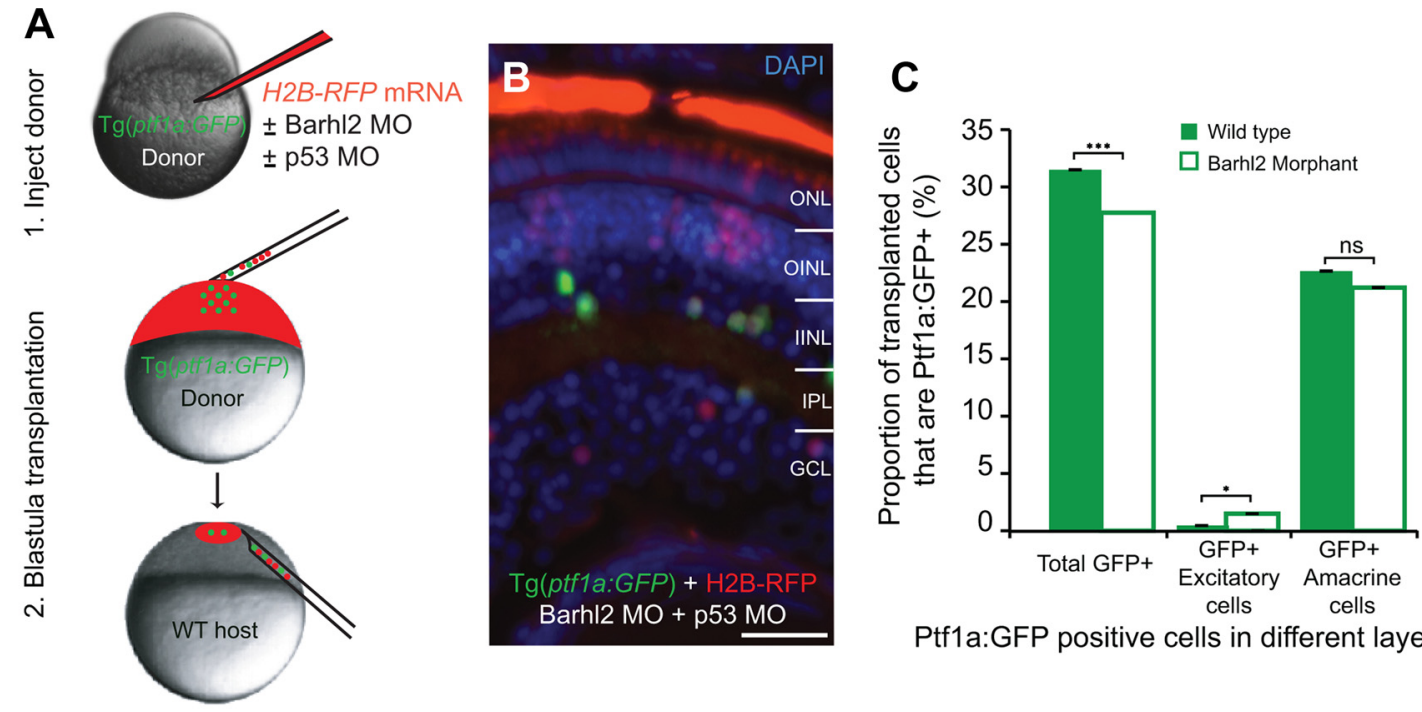

Ptf1a:GFP positive cells in different layers

\section{H2A-GFP donor cells into WT + DAPI}
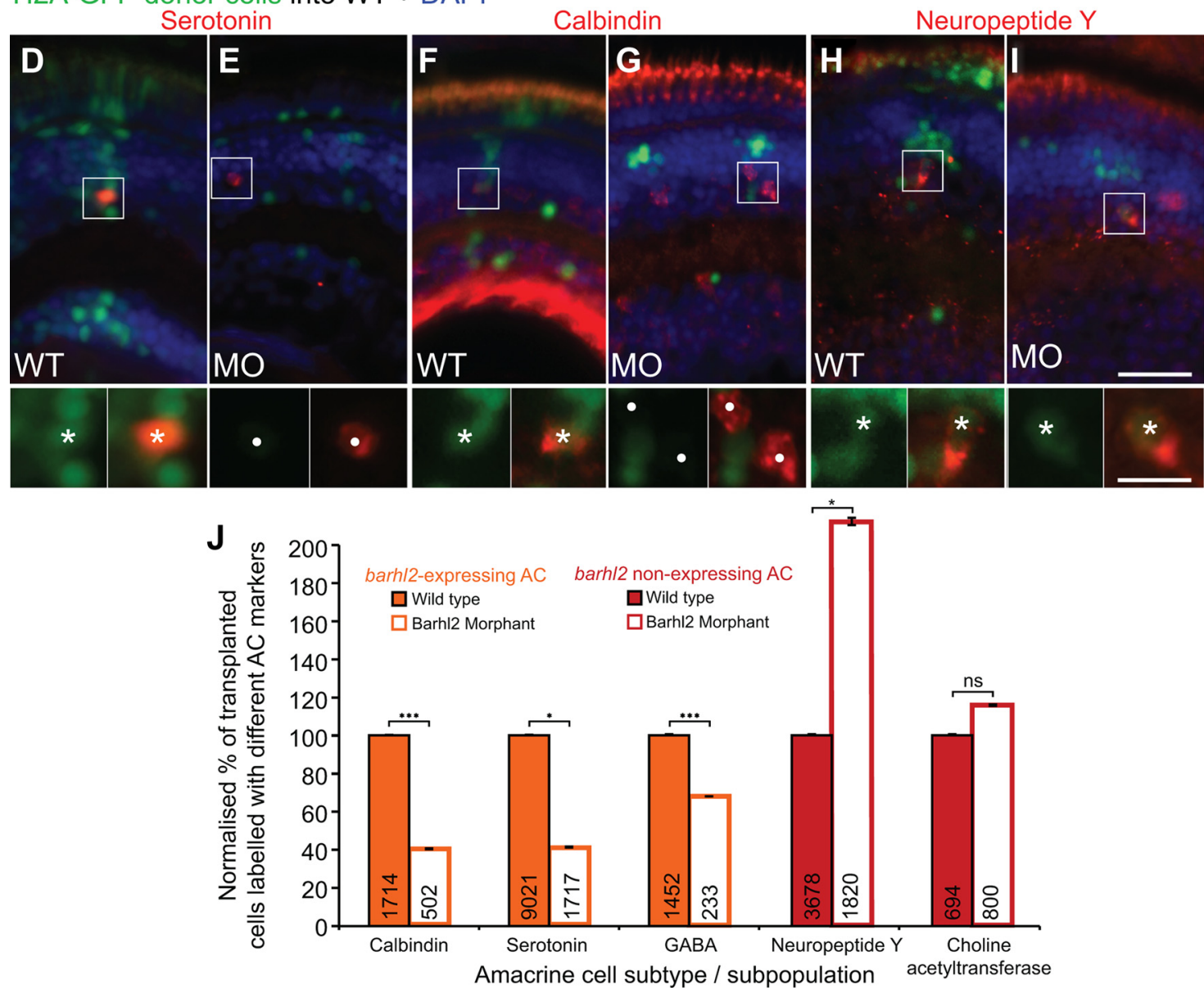

Figure 6. Barhl2 knockdown causes a subtype fate switch toward alternate inhibitory subtypes. $\boldsymbol{A}$, The cell-autonomous effect of Barhl2 loss was assessed with transplantations: Donor $\mathrm{Tg}$ (ptf1a:GFP) embryos were injected with H2B-RFP mRNA and p53 M0 (to aide cell survival; Robu et al., 2007), with (knockdown) or without (control) Barhl2 M0. Donor cells were subsequently transplanted into unlabeled WT. B, Some transplanted H2B-RFP cells express Ptf1a:GFP in both conditions. C, Quantification reveals a small reduction in the proportion of Ptf1a:GFP-labeled donor cells (32\% WT to 27.8\% morphant, $p<0.001$ ) and a few mislocalized Ptf1a:GFP cells in excitatory layers ( $0.74 \%$ WT to $1.49 \%$ morphants, $p=0.012)$. Overall, the vast majority of Ptf1a:GFP remain as inhibitory ACs (22.96\% WT to 21.2\% morphants, $p=0.13$ ). D-I, Immunohistochemically labeled amacrine subtypes (red) in chimeric retinas arise from transplanted H2A-GFP-labeled donor (e.g., asterisks for serotonin, $\boldsymbol{D}$; calbindin, $\boldsymbol{F}$; neuropeptide $Y, \boldsymbol{H}, \boldsymbol{I})$ and from unlabeled host cells (circles). J, Quantification of the proportion of labeled transplanted cells shows varying degrees of loss in subtypes that usually express barhl2 (orange) and increases in AC subtypes that usually do not (red). ONL, Outer nuclear layer; OINL, outer half of the inner nuclear layer; IINL, inner half of the inner nuclear layer; IPL, inner plexiform layer; GCL, ganglion cell layer; M0: morphant/morpholino; ns, not significant; ${ }^{*} p<0.05$; ${ }^{* * *} p<0.001$; error bars indicate SEM. Scale bars: $\boldsymbol{B}, 20 \mu \mathrm{m}$; (in I) $\boldsymbol{D}-\boldsymbol{I}, 25 \mu \mathrm{m}$; (in I inset) $\boldsymbol{D}-\boldsymbol{I}$ insets, $10 \mu \mathrm{m}$. 


\section{Tg(atoh7:gap43-RFP/barh/2:GFP)}
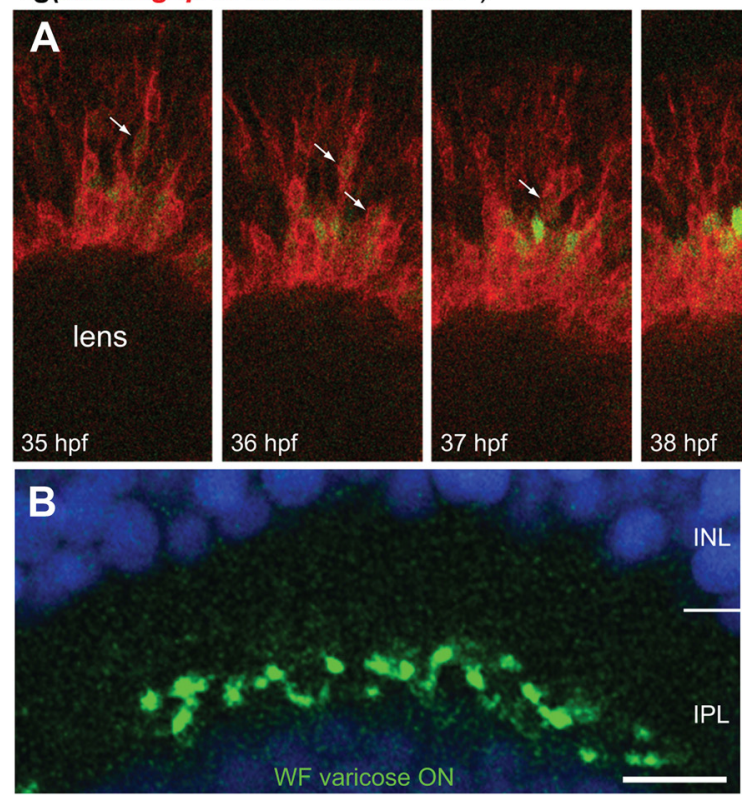
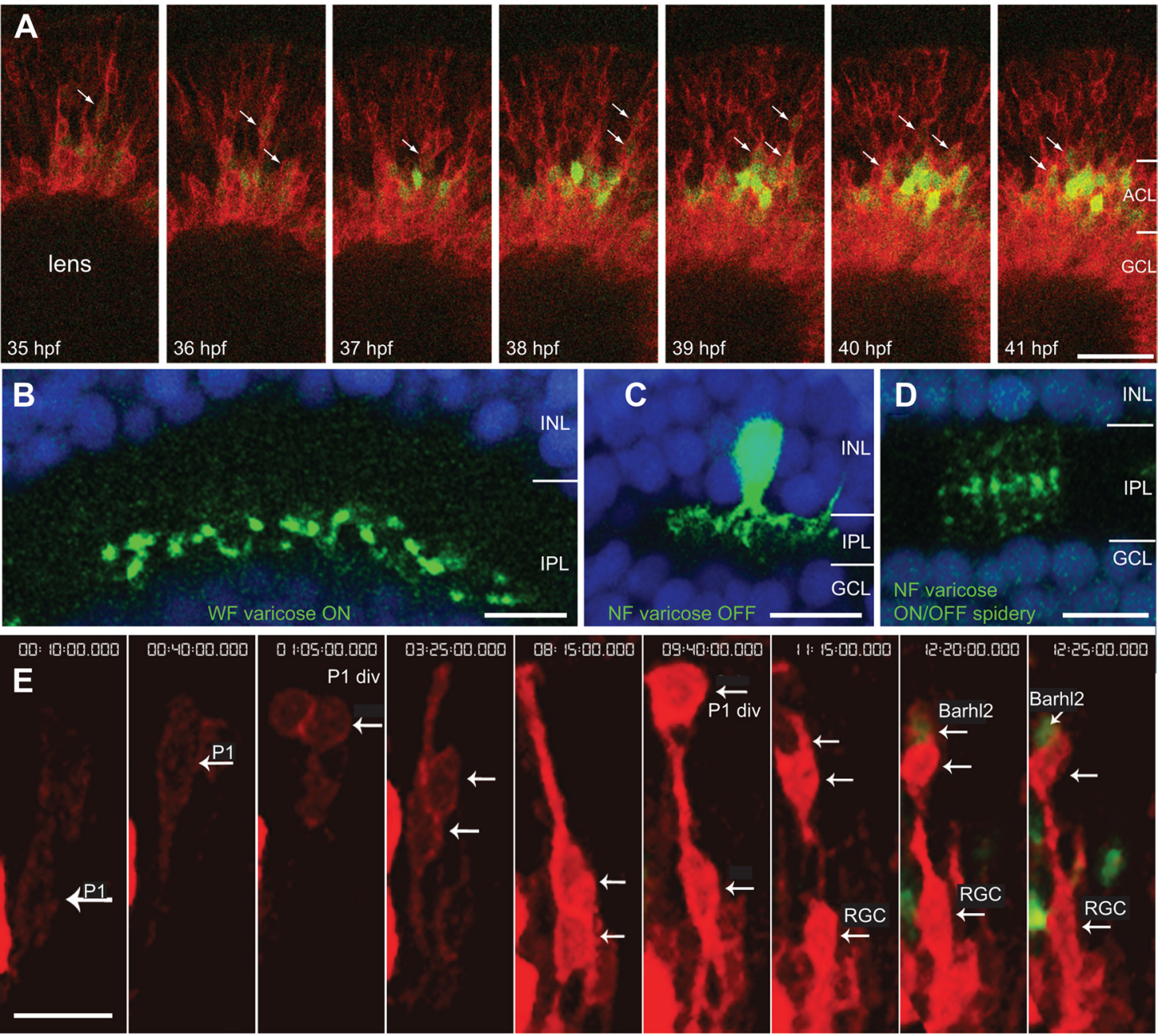

$\mathbf{F}$

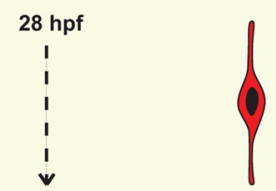

Atoh7 $\uparrow$

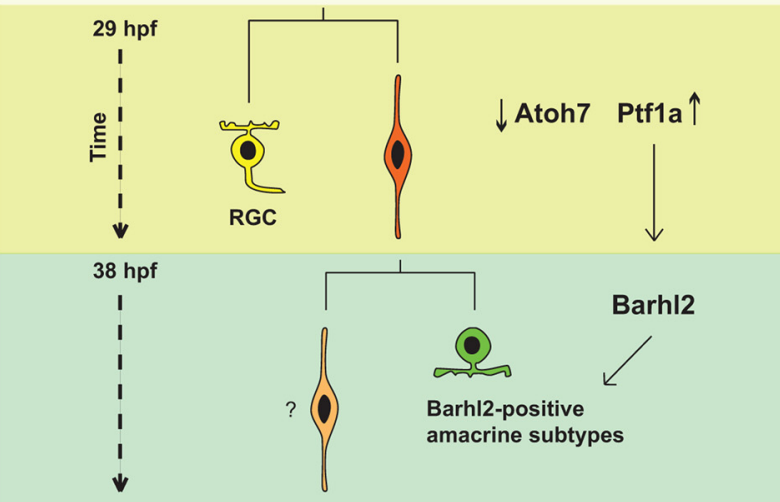

Figure 7. Barhl2 is expressed in nieces of atoh7-expressing progenitors. A, Micrographs from time-lapse movie ( $n=7$ movies) of double Tg(atoh7:gap43-RFP/barhl2:GFP) from 35 hpf. Barhl2:GFP cells almost always express Atoh7:gap43-RFP. Barhl2:GFP expression first occurs in cells that are migrating toward the amacrine layer. Boundaries are indicated for the forming amacrine $(\mathrm{ACL})$ and ganglion cell layer (GCL), which contains the brightly Atoh7:gap43-RFP-positive RGCs. Arrows indicate colabeling. Barhl2:GFP were analyzed from the first frame in which they appeared toward the center of the imaged stack (arrows). $\boldsymbol{B}-\boldsymbol{D}$, Examples of individual amacrine cells arising from barh/2 expression. Only one image of a confocal stack is shown, somas for some $\boldsymbol{B}$ and $\boldsymbol{D}$ were located at different depth. $\boldsymbol{E}$, Barhl2-positive cells derive from asymmetric divisions of RGC sisters in atoh7-expressing progenitors. 3D reconstruction of confocal stack from time-lapse series (starting at $28 \mathrm{hpf} t=0 \mathrm{~h}: 00 \mathrm{~min}$ ) shows the lineage of an Atoh7:gap43-RFP progenitor (P1). After P1 division (white arrow, P1 div, $t=1 \mathrm{~h}: 05 \mathrm{~min}$ ), both daughter cells migrate basally (white arrows, $t=$ $3 \mathrm{~h} 25 \mathrm{~min}$ ). At $t=8 \mathrm{~h} 15 \mathrm{~min}$, one daughter cell migrates apically, dividing at $t=9 \mathrm{~h} 40 \mathrm{~min}$ (white arrow, P2 div), whereas the other daughter cell remains close to the basal surface, differentiating as a RGC. At $t=12 \mathrm{~h} 20$ min one of the daughter cells from the P2 division upregulates Barhl2:GFP.F, Scheme summarizing the observed lineage tree and sequence of genes expression. Time is (Figure legend continues.) 


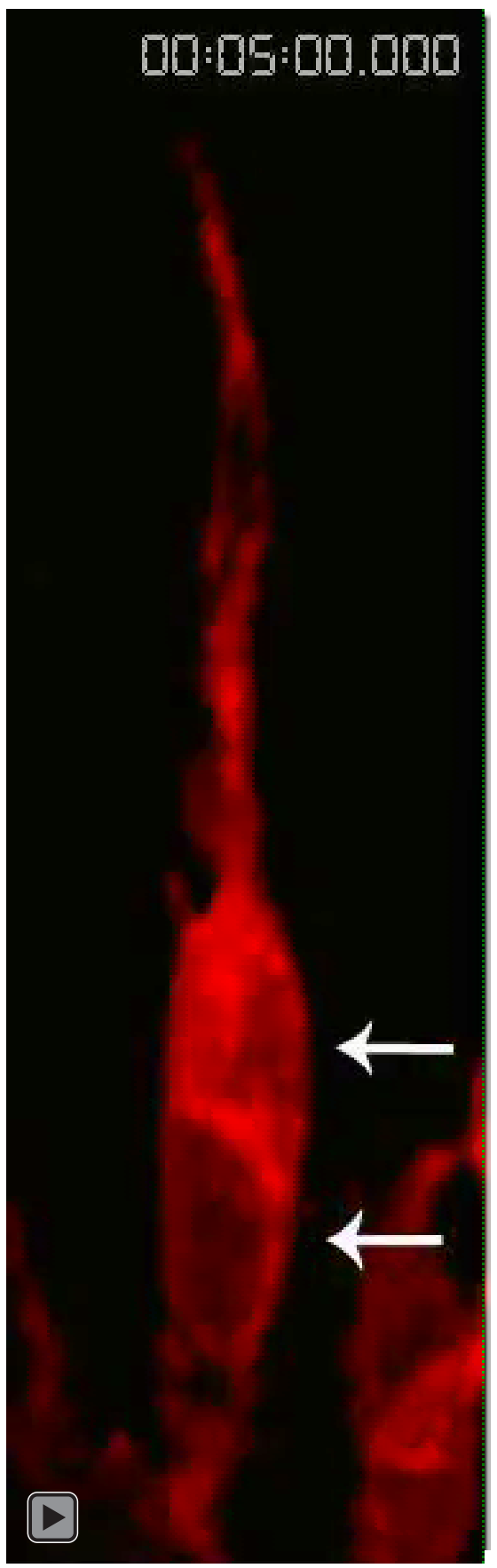

Movie 2. Time-lapse of developing retina showing the Barhl2:GFP cell generated from asymmetric cell division of an Atoh7:gap43-RFP progenitor. The apical surface is up, whereas the basal surface is down. The time-lapse begins with two sister cells (highlighted with white arrows, $t=0 \mathrm{h00} \mathrm{min}$ ) coming from an atoh7-expressing progenitor. One of them migrates back to the apical surface where it divides, generating one Barhl2:GFP cell, whereas the other sister remains close to the basal surface, differentiating as a RGC.

\section{Atoh7 affects lineage outcome and number of Barhl2-positive cells}

We know that ptf1a expression does not depend on Atoh7 (Jusuf et al., 2011), but as barhl2 is turned on in Atoh7-derived inhibi-

(Figure legend continued.) indicated on the left. Arrows indicate atoh7 being downregulated in the differentiating RGC and daughter cell while ptf1a is upregulated in the AC precursor (Jusuf and Haris, 2009; Jusuf et al., 2011; Brzezinski et al., 2012). NF, Narrow-field; WF, wide-field; P1, progenitor 1; P1 div, cell division of progenitor 1;P2 div, cell division of progenitor 2. Scale bars: $A, 30 \mu \mathrm{m} ; \boldsymbol{B}-\mathbf{D}, 10 \mu \mathrm{m} ; \boldsymbol{E}, 7 \mu \mathrm{m}$.

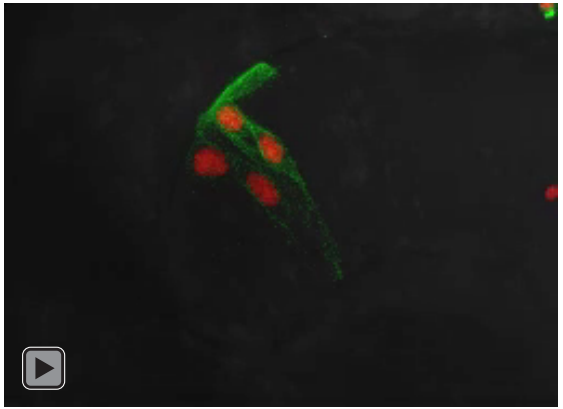

Movie 3. Time-lapse of developing retina showing premature barh/2 misexpression in atoh7-expressing progenitors. Time-lapse was performed from 28 to $40 \mathrm{hpf}$ ( $6 \mathrm{~min} /$ frame). In this transgenic zebrafish line $\mathrm{Tg}$ (atoh7:gal4/pUAS:gap43-GFP) injected with pUAS:barh/2-T2AH2B-RFP construct, misexpression of barh/2 (H2B-RFP-positive cells) prematurely in dividing Atoh7-positive progenitors apically located was confirmed.

tory precursors, we wondered whether Atoh7 influences the way Barhl2-positive cells arise within the Atoh7-lineage. The Tg(barhl2:GFP) line was outcrossed to atoh $7^{-1-}$ (lakritz) mutants (Kay et al., 2001). Barhl2:GFP expression analyzed at $5 \mathrm{dpf}$ is not only retained, but also virtually doubled in the absence of Atoh7 $(39.33 \pm 0.92 \%$ SEM WT to $73.74 \pm 2.261 \%$ SEM lakritz, $p<$ 0.0001 ; data not shown) with significant increases in the INL $(48.33 \pm 1.32 \%$ SEM to $78.32 \pm 2.35 \%$ SEM, $p<0.0001$; data not shown $)$ and GCL $(20 \% \pm 1.03$ SEM to $63.89 \pm 3.81 \%$ SEM, $p<$ 0.0001; data not shown). How do Barhl2-positive ACs arise in this case? In 12 time-lapse movies of Barhl2:GFP/Atoh7:gap43RFP cells in lakritz retinas, we consistently observe Atoh7:gap43RFP-positive cell divisions generating two daughters, only one of which becomes Barhl2:GFP-positive. Interestingly, in eight of these movies, the original Atoh7:gap43-RFP cells first divide once as usual. However, as neither daughter is able to differentiate into a RGC, both daughters divide again, with one cell from each pair starting to express Barhl2:GFP (Movie 4). Thus, when Atoh7 is missing, Barhl2-positive ACs still arise within asymmetric cell divisions from the "Atoh7-lineage," but in this case there are two rather than only one barhl2-expressing cell generated from each lineage. This different lineage-outcome reveals the mechanism of Barhl2-positive amacrine cell's increased number in the lakritz mutant.

Atoh7 affects Barhl2-positive amacrine subtypes identity Are barhl2-expressing subtypes increased equally in the absence of Atoh7? Since GABA labels the majority of Barhl2:GFP cells, we first assessed changes to this cell population. We found that the total proportion of Barhl2:GFP/GABAergic neurons remains unchanged in lakritz mutants (32\% WT to $30 \%$ lakritz). Because GABAergic neurons consist of multiple subtypes, we next assessed, if specific subtypes are changed within this population. We found that serotonergic amacrine subtypes (usually barhl2expressing) are significantly increased $(+20.6 \pm 6 \%$ SEM lakritz retina, $p=0.0002$, Fig. $9 E-G)$, thus suggesting that specific barhl2-expressing subtypes are preferentially affected. The most striking change is the large increase in Barhl2:GFP non-GABAergic amacrine cells in the lakritz retina (Fig. 9C,D). One possible explanation for this outcome could be that Barhl2-negative subtypes now become Barhl2-positive. To investigate on this possibility, we analyzed the population of NY+ amacrine subtypes. We found no significant change in NY+ proportion or GFP expression $(+$ or $-11 \% \pm 5.5$ SEM lakritz retina, $p=$ 0.2611 ). Our data thus show that specific subtypes of Barhl2: 
A

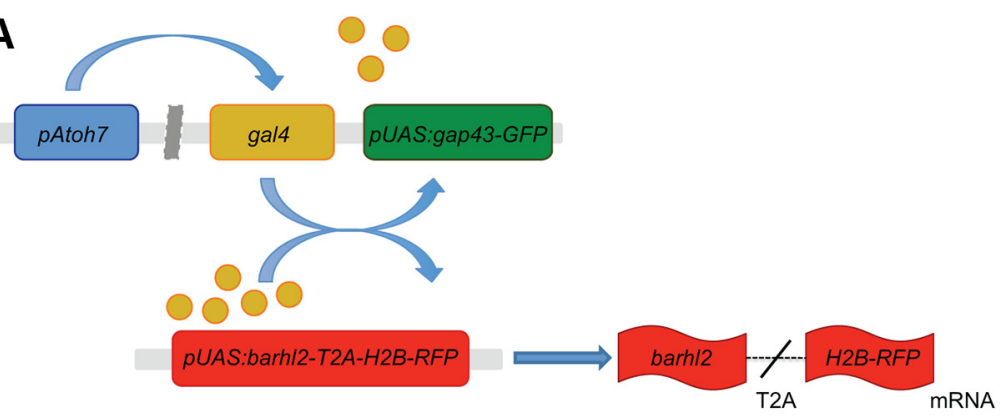

\section{B Control}

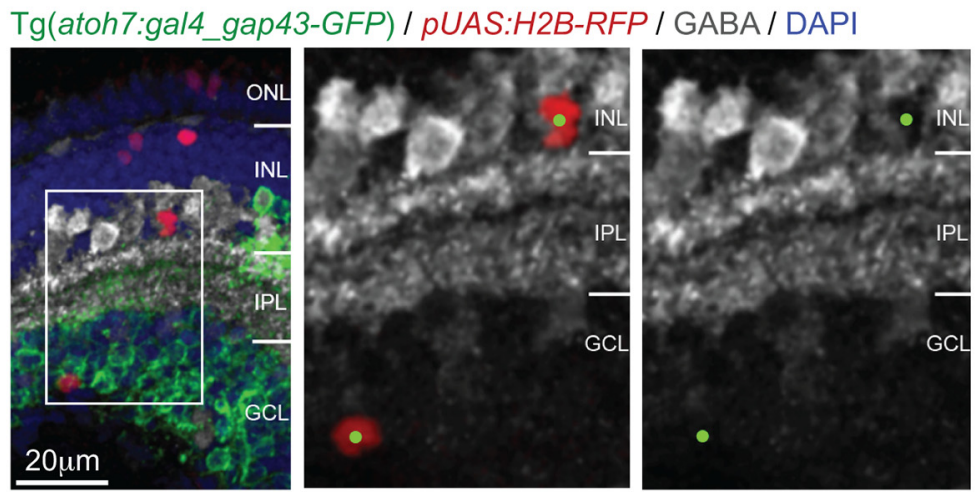

\section{Misexpression}

Tg(atoh7:gal4_gap43-GFP) / pUAS:barh/2
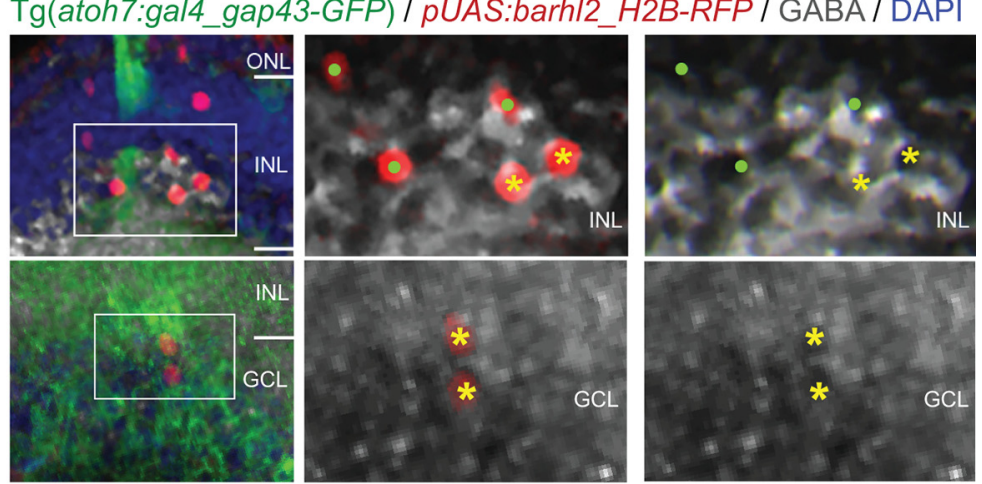

D

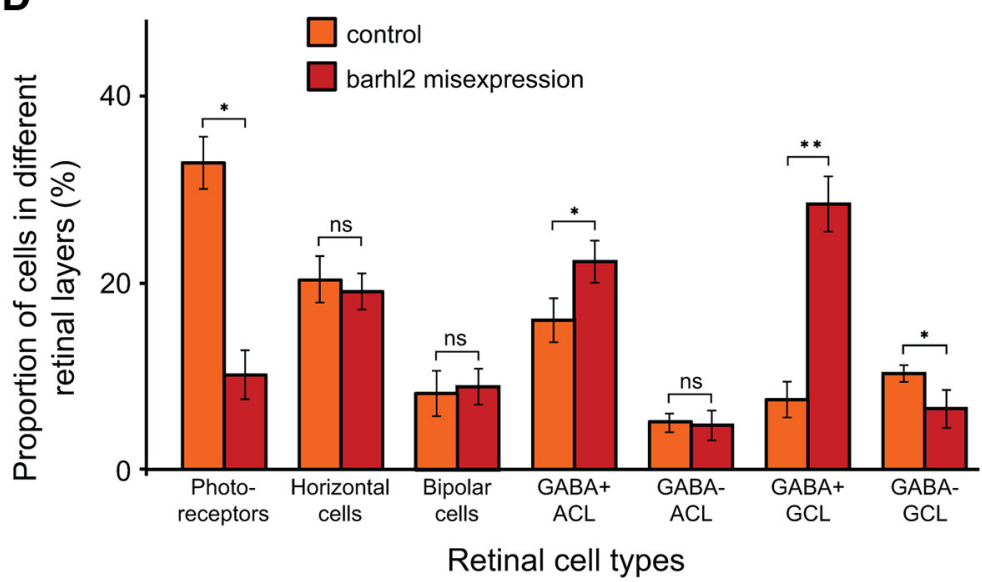

Figure 8. Misexpression of barh/2 in atoh7-expressing progenitors drives the fate of amacrine subtypes that usually express barh12. A, Schematic showing misexpression design: The atoh7 promoter drives expression of Gal4 transcription factor. Gal4
GFP + cells, including 5-HT + and nonGABAergic populations, are preferentially expanded. Although the increase in amacrine cells can be explained by our lineage analysis, the mechanism by which Atoh7 affects subtype identity remains unclear.

Lakritz mutants retain some cells in the ganglion cell layer of the retina, which have been attributed to displaced amacrine cells (Kay et al., 2001). Does this simply reflect the tendency of ACs to occupy the now RGC-free most basal positions in the retina (Kay et al., 2001), or rather it indicates a selective increase in specific subclasses of amacrine cells (Feng et al., 2010), e.g., the Barhl2:GFP cells deriving from the Atoh7-lineage? To gain more insights into this question we analyzed GABA as marking a large proportion of the usually Barhl2 + population, as well as serotonin and neuropeptide $\mathrm{Y}$ as subtype-specific markers that were usually Barhl2 + or Barhl2-, respectively. We found GABAergic Barhl2:GFP increased in the GCL $(12.8 \%$ WT to $21 \%$ lakritz, $p=$ $0.0008)$ and decreased in the INL $(19.2 \%$ WT to $9 \%$ lakritz, $p=0.0014$ ) (Fig. $9 A-$ D). Similarly, serotonergic Barhl2:GFP cells, which are normally only found in the INL (Fig. 9E-G) are now increased due to their appearance in the GCL $(+20.2 \%$ increase, $p=0.0002$, Fig. $9 E-$ $G)$. Finally, even though the proportion of $\mathrm{NY}+$ remained unchanged in the lakritz retina, some of these cells were redistributed to the GCL $(11 \%$ increase in the lakritz retina compared with the WT, $p=$ 0.0009 , data not shown). These observations together suggest that amacrine sub-

\section{$\leftarrow$}

activates the upstream activation sequence ( $p U A S$ ) promoter to drive expression of gap43-GFP reporter by itself (control) or with Barhl2 and H2B-RFP reporter generated through in frame fusion with T2A peptide. After translation of the barhl2-T2AH2B-RFPmRNA the T2A sequence will be cleaved and generate separate Barhl2 and H2B-RFP proteins. B, C, Micrographs of $120 \mathrm{hpf} \mathrm{Tg}$ (atoh7:gal4/pUAS:gap43-GFP) retinas with pUAS driving H2B-RFP (control) or barhl2 and H2B-RFP (misexpression), and subsequently labeled for GABA (white). Boxes indicate higher-power insets. Cell type and GABA colabeling of H2B-RFP-expressing cells was analyzed. Examples of GABApositive cells (yellow asterisks) and GABA-negative cells (green dots). D, Quantification of cell fates in control (orange) or barhl2 misexpression (red). There is an increase in GABAergic cells in the INL $(15.93 \pm 4.86 \%$ SEM control to $22.2 \pm$ 4.64\% SEM misexpression, $p=0.0044$ ) and particularly GCL (7.5 $\pm 4 \%$ SEM control to $28.32 \pm 6.05 \%$ SEM misexpression, $p<0.0001$ in $\mathrm{GCL}$ ) with a concurrent loss in mainly photoreceptors (32.84 $\pm 5.76 \%$ SEM control to $10.12 \pm 5.4 \%$ SEM misexpression, $p=0.01058$ ) and presumed ganglion cells. ONL, Outer nuclear layer; IPL, inner plexiform layer; GCL, ganglion cell layer; ns, not significant. Error bars indicate SEM. ${ }^{*} p<0.05,{ }^{* *} p<0.001$. Scale bar (in $\left.B\right) B, C, 20 \mu \mathrm{m}$. 

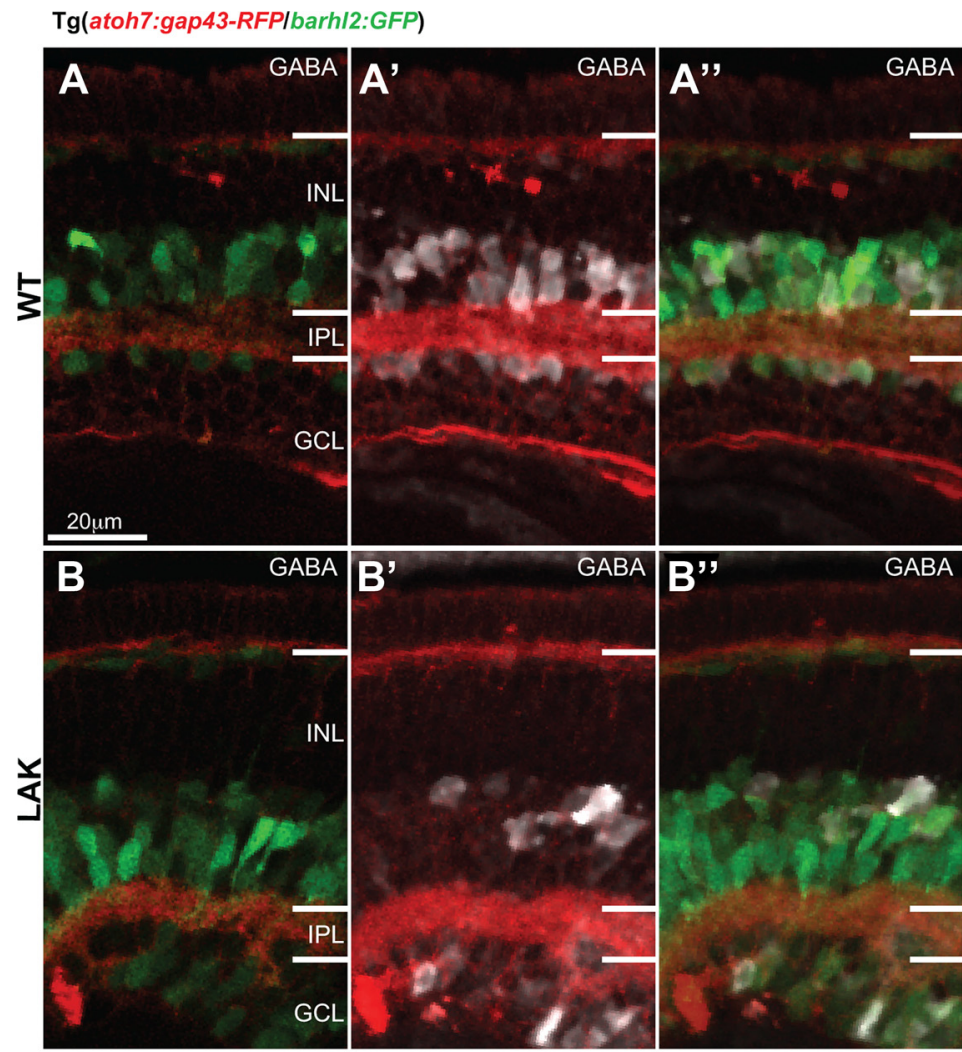

GABA
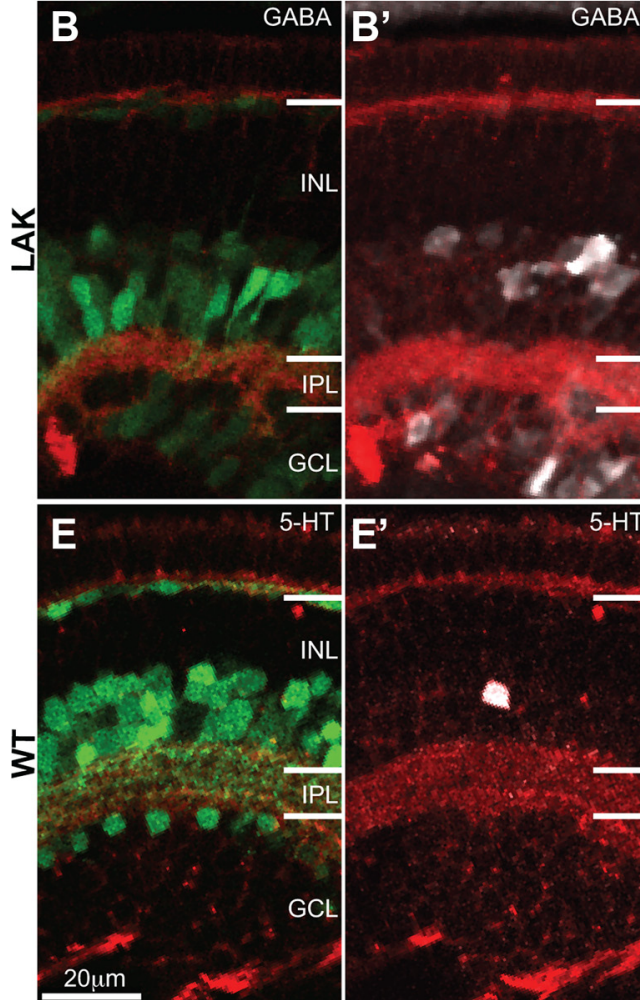

$B^{\prime \prime}$

GABA
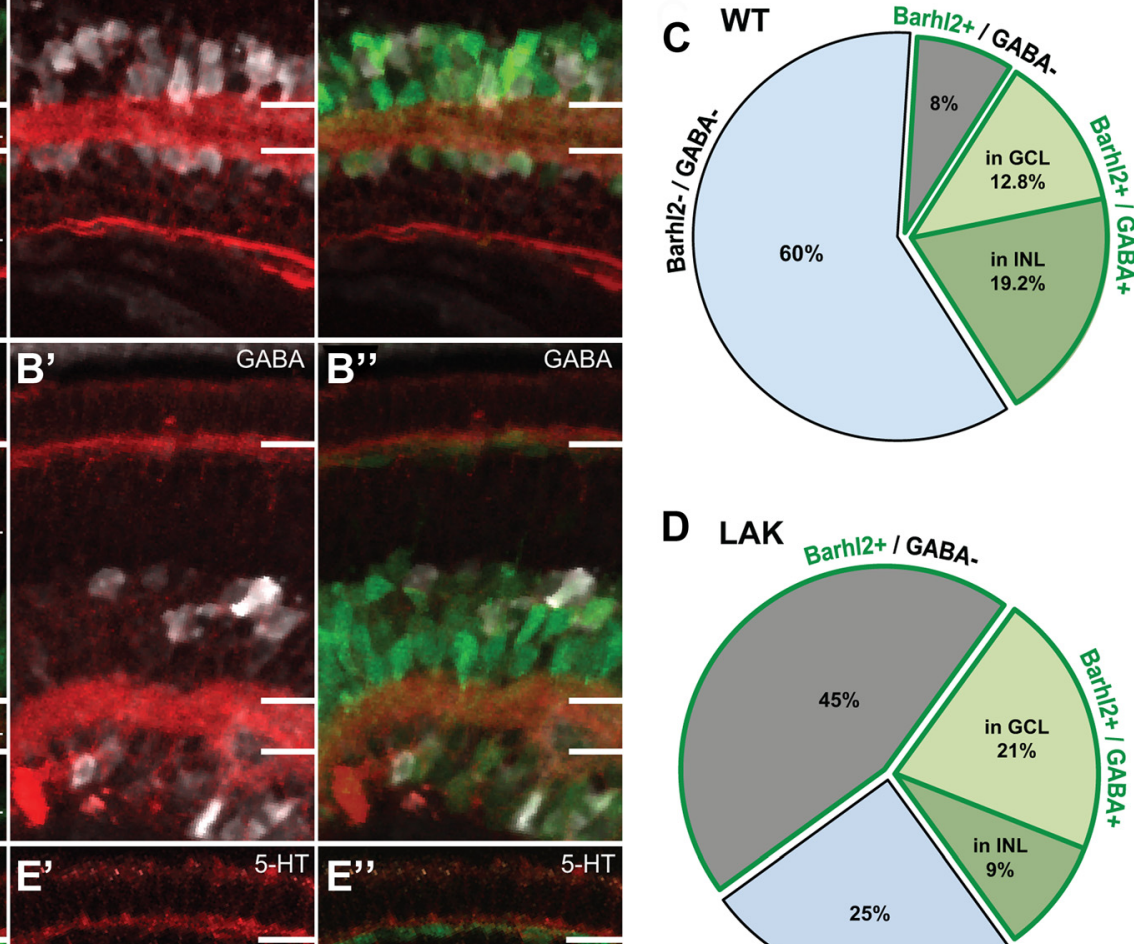

D LAK
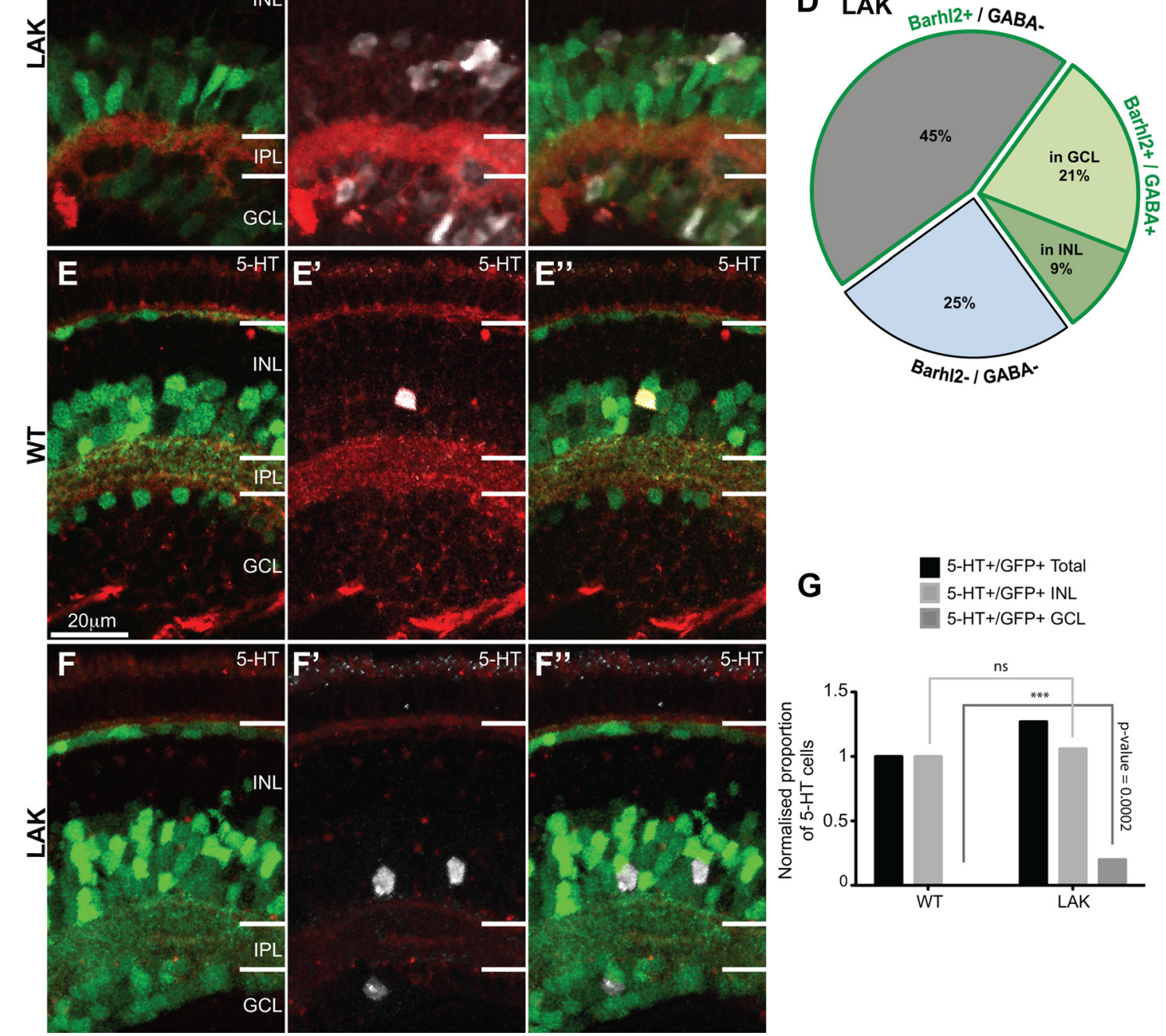

Figure 9. Absence of Atoh7 leads to an increase of barh/2-expressing ACs in the ganglion cell layer. A, B, Micrographs of $120 \mathrm{hpf}$ Tg(atoh7:gap43-RFP/barh/2:GFP) retinas labeled for GABA in WT $(\boldsymbol{A})$ and lakritz (LAK) (B).C, D, Quantification of amacrine subpopulations expressed as proportion of total INL and GCL cells. The data indicate that the increase in the number of GFP + cells in the LAK retina is primarily due to non-GABAergic ACs, as the proportion of GABAergic ACs remains unchanged in total in both GCL and INL in LAK versus WT (32\% WT to $30 \%$ lakritz). A redistribution of GABAergic ACs is, however, observed with an increase of Barhl2 +/GABA + cells in the GCL and a relative decrease of those cells in the INL of the LAK retina compared with WT (12.8\% WT to 21\% LAK, $p=0.0008$ in GCL and 19.2\% WT to 9\% LAK, $p=0.0014$ in INL). Total number of cells is the number of nuclei in the INL and GCL. E, F, Micrographs of 120 hpf Tg(atoh7:gap43-RFP/barhl2:GFP) retinas labeled for serotonin (5-HT). G, Quantification of the total proportion of serotonin-positive/Barhl2:GFP-positive (5-HT+/GFP+) neurons shows an increase in 5-HT cells in lakritz specifically in the $\mathrm{GCL}$ where they are normally absent $\left(n=15-19\right.$ eyes). IPL, Inner plexiform layer; $\mathrm{GCL}$, ganglion cell layer. ${ }^{* * *} p<0.0002$. Error bars indicate SEM. Scale bars, $20 \mu \mathrm{m}$. 


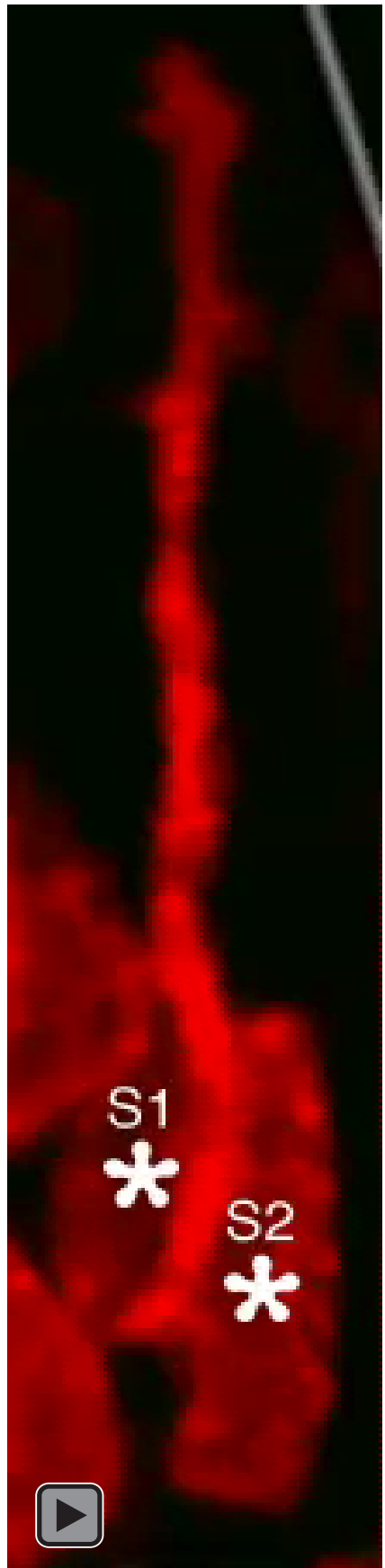

Movie 4. Time-lapse of developing retina showing the Barhl2:GFP-positive cells derived from asymmetric divisions of two sister cells expressing Atoh7:gap43-RFP in the lakritz (atoh $7^{-1-}$ ) mutant. The apical surface is up, whereas the basal surface is down. Every frame corresponds to $5 \mathrm{~min}$ of the time-lapse. The time-lapse begins with two sister cells (S1 and S2, highlighted with asterisks) coming from an atoh7-expressing progenitor. The S1 cell (Sister cell 1) migrates toward the apical surface to divide asymmetrically, generating one Barhl2:GFP-positive (S1a) and one Barhl2:GFP-negative cell (S1b). The S2 cell (Sister cell 2) also migrates toward the apical surface to divide asymmetrically, $\sim 3-4 \mathrm{~h}$ after the cell division of Sister cell 1. The division of Sister cell 2 also generates one Barhl2:GFP-positive (S2a) and one Barhl2:GFP-negative cell (S2b).

types get redistributed to the ganglion cell layer in the lakritz mutants, regardless of whether they are respecified or increased in number.

\section{Discussion}

Although neural cell fate determination factors are being studied in various model organisms, the occurrence of particular patterns and interactions within individual cell lineages can only be assessed in vivo in the zebrafish model system. In this study we use a combination of functional and in vivo time-lapse analyses to uncover novel aspects of lineage-related transcriptional networks and cell-fate outcomes in the developing vertebrate retina. In particular, we here demonstrate that the zebrafish Barhl2 paralog biases particular ACs in vivo, and is turned on in particular Atoh7-lineages.

In the zebrafish retina, $71 \%$ of inhibitory neurons (all horizontal and $\sim 60 \%$ of amacrine cells) derive from Atoh7 progenitors. These are distinct in subtype composition from the remaining third of amacrine subtypes that derive from nonAtoh7 progenitors (Jusuf et al., 2011). Most amacrine subtypes can arise from either progenitor, but the probability of assuming a particular subtype fate correlates with whether the AC arose from an Atoh7-positive or -negative lineage. Experimentally induced coexpression of $p t f 1 a$ in all Atoh7-positive progenitors leads to a selective increase in the ACs that normally derive from the Atoh7-lineage (Jusuf et al., 2011). Yet Atoh7 is not essential for the specification of any of the amacrine subtypes, and $p t f 1 a$ is expressed in all ACs, suggesting that other factors must be key to biasing subtype specification. Here we find that $58.5 \%$ of amacrine cells express barhl2, and that most Barhl2 cells arise from atoh7-expressing progenitors, thus providing evidence that Barhl2 may be this "other" factor. Consistent with this hypothesis, we found that barhl2-expressing subtypes were similarly underrepresented and overrepresented when compared with our previously characterized Atoh7+/Ptf1a + lineage. Furthermore, knocking down or increasing barhl2 expression within Atoh7progenitors is sufficient to bias the differentiation of amacrine cells in predictable directions. Together these observations provide an explanation as to why the $p t f 1 a$-expressing progenitors in the Atoh7-lineage, but not those from the non-Atoh7 lineage, bias differentiation toward specific amacrine subtypes and not others (Jusuf et al., 2011).

Barhl2 has been implicated in the development of ACs, and specification and survival of RGCs downstream of atoh7 in the mouse and Xenopus retina (Mo et al., 2004; Poggi et al., 2004; Wang and Harris, 2005; Ding et al., 2009). Here we find that the zebrafish barhl2 paralog is turned on exclusively in postmitotic, inhibitory Ptfla-positive ACs and horizontal cells. We also find that barhl2 is preferentially expressed at different levels in specific amacrine subtypes (such as GABA, 5-HT and calbindin expressing), whereas it is almost completely absent in others (NY and ChAT). In accordance with data from mouse, the absence of Barhl2 function leads to an altered amacrine subtype composition (Ding et al., 2009). Specifically, subtypes that are normally Barhl2-positive (GABA, serotonin and calbindin) decrease at the expense of the ones that normally do not express barhl2 (ChAT and NY). Decreases in GABAergic population with increases in cholinergic AC are consistent with the previous mouse data (Ding et al., 2009) and these may indeed represent homologous subtypes. However, other subtypes may present preferential alternate fates, as the proportional increase in NY cells far outweighed the modest and statistically not significant increase in ChAT + cells. Although some of the markers may label overlapping amacrine subtype populations, our results show specific losses in subtypes that express barhl2 most strongly. We therefore show for the first time a strong direct correlation between expression levels of barhl2 and cell-autonomous necessity for Barhl2 in each of these specific subtypes, rather than just a general role in relative subtype composition.

Unlike in other vertebrates (Poggi et al., 2004; Ding et al., 2009), the zebrafish barhl2 is not likely implicated in RGC differ- 
entiation or survival. Several observations support this conclusion: (1) barhl2 expression is restricted to ptfla-expressing inhibitory cell precursors and is turned on only after the division of sister cell of an RGC; (2) Overexpression of barhl2 is sufficient for amacrine subtypes, but not RGCs, even if overexpressed prematurely under the control of the atoh7 promoter; (3) knocking down barhl2 does not show significant changes in RGC genesis; (4) barhl2 expression depends on the expression of ptfla and not on the expression of atoh7. All of these observations are in agreement with Barhl2 acting exclusively in inhibitory cell precursors. The function of barhl genes in RGCs genesis or maintenance might instead be retained by the duplicated barhl1.2 paralog; which is expressed in RGCs downstream of Atoh7 (Schuhmacher et al., 2011).

What is then the relationship between barhl2 and atoh7? Previous expression analysis suggested that a negative feedback might occur between the two genes, possibly as a result of the conservation of an ancestral feature existing in the invertebrate Drosophila melanogaster between the homologues atonal and barH genes (Lim and Choi, 2003; Schuhmacher et al., 2011). Our in vivo time-lapse study highlighted particular lineagerelationships between Atoh7-dependent RGCs and barhl2expressing amacrine subtypes. Our results clearly demonstrate that the expression of the two genes is mutually exclusive at cellular level; they also show that Barhl2-positive ACs arise as nieces of RGCs, which depend on Atoh7. This observation, combined with results from loss- and gain-of-function assigns the timing of Barhl2 action in postmitotic precursors in which atoh 7 has been already downregulated (Skowronska-Krawczyk et al., 2005; Le et al., 2006; Brzezinski et al., 2012) and the switch from RGC to inhibitory cell fates has already occurred via the expression of ptfla. Thus, Barhl2 in vivo is not well positioned to act as a feedback repressor of atoh7. The intermediate ptfla expression however, might in fact repress atoh 7 within this lineage consistent with data in chick (Lelièvre et al., 2011; Fig. 7F).

Conversely, we found that barhl2 expression does not need functional Atoh7. Atoh7 might therefore exert an indirect repressive function on barhl2. A recent study in the mouse shows that in Math5/atoh7-null retina there is a precocious expression of AC fate determinants, thus suggesting that Atoh7 prevents ACs from being generated prematurely (Feng et al., 2010). Yet our in vivo time-lapse analysis of barhl2:GFP/atoh7:gap43-RFP progenitors through two rounds of cell division shows that the onset of Barhl2:GFP within individual cell lineages is unvaried in the absence of Atoh7. Consistently, in both wild-type and lakritz retina, Barhl2-dependent ACs tend to arise from divisions of "Atoh7: gap43-RFP" cells in which one daughter turns on Barhl2:GFP and the other does not. However, we also find that Atoh7 affects the lineage-outcome of retinal progenitor cells. Therefore, the increase in the number of Barhl2-positive cells is rather due to the fact that without Atoh7, both daughters of one Atoh7:gap43-RFP progenitor divide again to generate an additional Barhl2-positive daughter. This is coherent with a co-temporaneous study by $\mathrm{He}$ et al. (2012 in press), in which randomly selected lineages in the zebrafish retina were traced in time-lapse. In these lineages, $\sim 80 \%$ of all RGCs arise from asymmetric divisions in which one daughter differentiates as an RGC and the other daughter divides again. Results from $\mathrm{He}$ et al. also show that in the absence of Atoh7, there is a marked decrease in such divisions.

Although the onset of barhl2 is independent of Atoh7 action, our results clearly show that Atoh7 does affect barhl2-expressing amacrine subtypes identity. While GABAergic/Barhl2-positive amacrines remain unchanged in the lakritz retina, there is a strik- ing increase in the non-GABAergic/Barhl2-positive cells and in the normally rare serotonin/Barhl2-positive ACs. Thus, Atoh7 might be permissive for some Barhl2-dependent amacrine subtypes (such as GABA) while it might repress others (e.g., serotonin). In the atoh7-null retinas of the mouse, a change in amacrine subtype composition has also been noted, with certain amacrines increasing at the expense of others (Feng et al., 2010). Also in agreement with these studies, our analysis of the lakritz retina shows an overall increase in displaced ACs (Kay et al., 2001; Feng et al., 2010). This general increase was happening regardless of whether ACs where particular Barhl2-positive or Barhl2negative (such as for NY) subtypes.

It remains a major challenge to identify cell fate determination networks and how they operate within the context of lineage in the developing vertebrate CNS. Recent studies suggest that there are stochastic mechanisms at work in determining fate choice within clones (Gomes et al., 2011; He et al., 2012), and perhaps that is why factors like Barhl2 bias rather than strictly determine fate choice, but our results showing the rather strict expression of Barhl2 within theAtoh7-lineage argue that the expression of at least some cellular determinants in the retina are strongly influenced lineage. We have shown that the zebrafish retina is an excellent model system to address this challenge as this system allows us to follow in vivo modes of cell divisions within individual lineages, concurrently with the expression of specific cell fate determinants. Here, we highlighted the lineage of individual atoh7-expressing progenitors through two rounds of cell divisions, and our functional results suggest that interactions involving the division mode, and the transcriptional network of Atoh7, Ptfla and Barhl2 promote the orderly generation of RGCs and amacrine subtypes within this lineage.

\section{References}

Brown NL, Patel S, Brzezinski J, Glaser T (2001) Math5 is required for retinal ganglion cell and optic nerve formation. Development 128:2497-2508.

Brzezinski JA 4th, Kim EJ, Johnson JE, Reh TA (2011) Ascll expression defines a subpopulation of lineage-restricted progenitors in the mammalian retina. Development 138:3519-3531.

Brzezinski JA 4th, Prasov L, Glaser T (2012) Math5 defines the ganglion cell competence state in a subpopulation of retinal progenitor cells exiting the cell cycle. Dev Biol 365:395-413.

Clemente D, Porteros A, Weruaga E, Alonso JR, Arenzana FJ, Aijón J, Arévalo R (2004) Cholinergic elements in the zebrafish central nervous system: histochemical and immunohistochemical analysis. J Comp Neurol 474: 75-107.

Ding Q, Chen H, Xie X, Libby RT, Tian N, Gan L (2009) BARHL2 differentially regulates the development of retinal amacrine and ganglion neurons. J Neurosci 29:3992-4003.

Dullin JP, Locker M, Robach M, Henningfeld KA, Parain K, Afelik S, Pieler T, Perron M (2007) Ptfla triggers GABAergic neuronal cell fates in the retina. BMC Dev Biol 7:110.

Feng L, Xie ZH, Ding Q, Xie X, Libby RT, Gan L (2010) MATH5 controls the acquisition of multiple retinal cell fates. Mol Brain 3:36.

Fujitani Y, Fujitani S, Luo H, Qiu F, Burlison J, Long Q, Kawaguchi Y, Edlund H, MacDonald RJ, Furukawa T, Fujikado T, Magnuson MA, Xiang M, Wright CV (2006) Ptfla determines horizontal and amacrine cell fates during mouse retinal development. Development 133:4439-4450.

Ghiasvand NM, Rudolph DD, Mashayekhi M, Brzezinski JA 4th, Goldman D, Glaser T (2011) Deletion of a remote enhancer near ATOH7 disrupts retinal neurogenesis, causing NCRNA disease. Nat Neurosci 14:578-586.

Godinho L, Mumm JS, Williams PR, Schroeter EH, Koerber A, Park SW, Leach SD, Wong RO (2005) Targeting of amacrine cell neurites to appropriate synaptic laminae in the developing zebrafish retina. Development 132:5069-5079.

Gomes FL, Zhang G, Carbonell F, Correa JA, Harris WA, Simons BD, Cayouette M (2011) Reconstruction of rat retinal progenitor cell lineages in vitro reveals a surprising degree of stochasticity in cell fate decisions. Development 138:227-235. 
He JC, Zhang G, Almeida AD, Cayouette M, Simons BD, Harris WA (2012) How variable clones build an invariant retina. Neuron 75:739-742.

Holt CE, Bertsch TW, Ellis HM, Harris WA (1988) Cellular determination in the Xenopus retina is independent of lineage and birth date. Neuron $1: 15-26$.

Juraver-Geslin HA, Ausseil JJ, Wassef M, Durand BC (2011) Barhl2 limits growth of the diencephalic primordium through Caspase3 inhibition of beta-catenin activation. Proc Natl Acad Sci U S A 108:2288-2293.

Jusuf PR, Harris WA (2009) Ptfla is expressed transiently in all types of amacrine cells in the embryonic zebrafish retina. Neural Dev 4:34.

Jusuf PR, Almeida AD, Randlett O, Joubin K, Poggi L, Harris WA (2011) Origin and determination of inhibitory cell lineages in the vertebrate retina. J Neurosci 31:2549-2562.

Kawakami K (2004) Transgenesis and gene trap methods in zebrafish by using the Tol2 transposable element. Methods Cell Biol 77:201-222.

Kay JN, Finger-Baier KC, Roeser T, Staub W, Baier H (2001) Retinal ganglion cell genesis requires lakritz, a Zebrafish atonal Homolog. Neuron 30:725-736.

Kim JH, Lee SR, Li LH, Park HJ, Park JH, Lee KY, Kim MK, Shin BA, Choi SY (2011) High cleavage efficiency of a 2A peptide derived from porcine teschovirus-1 in human cell lines, zebrafish and mice. PLoS One 6:e18556.

Kimmel CB, Ballard WW, Kimmel SR, Ullmann B, Schilling TF (1995) Stages of embryonic development of the zebrafish. Dev Dyn 203:253-310.

Kinkhabwala A, Riley M, Koyama M, Monen J, Satou C, Kimura Y, Higashijima S, Fetcho J (2011) A structural and functional ground plan for neurons in the hindbrain of zebrafish. Proc Natl Acad Sci U S A 108:1164-1169.

Le TT, Wroblewski E, Patel S, Riesenberg AN, Brown NL (2006) Math5 is required for both early retinal neuron differentiation and cell cycle progression. Dev Biol 295:764-778.

Lelièvre EC, Lek M, Boije H, Houille-Vernes L, Brajeul V, Slembrouck A, Roger JE, Sahel JA, Matter JM, Sennlaub F, Hallböök F, Goureau O, Guillonneau X (2011) Ptf1a/Rbpj complex inhibits ganglion cell fate and drives the specification of all horizontal cell subtypes in the chick retina. Dev Biol 358:296-308.

Lim J, Choi KW (2003) Bar homeodomain proteins are anti-proneural in the Drosophila eye: transcriptional repression of atonal by Bar prevents ectopic retinal neurogenesis. Development 130:5965-5974.

Lin JW, Biankin AV, Horb ME, Ghosh B, Prasad NB, Yee NS, Pack MA, Leach SD (2004) Differential requirement for ptfla in endocrine and exocrine lineages of developing zebrafish pancreas. Dev Biol 274:491-503.

Mo Z, Li S, Yang X, Xiang M (2004) Role of the Barhl2 homeobox gene in the specification of glycinergic amacrine cells. Development 131:1607-1618.

Nakhai H, Sel S, Favor J, Mendoza-Torres L, Paulsen F, Duncker GI, Schmid RM (2007) Ptfla is essential for the differentiation of GABAergic and glycinergic amacrine cells and horizontal cells in the mouse retina. Development 134:1151-1160.

Pittman AJ, Law MY, Chien CB (2008) Pathfinding in a large vertebrate axon tract: isotypic interactions guide retinotectal axons at multiple choice points. Development 135:2865-2871.

Poggi L, Vottari T, Barsacchi G, Wittbrodt J, Vignali R (2004) The homeobox gene Xbh1 cooperates with proneural genes to specify ganglion cell fate within the Xenopus neural retina. Development 131:2305-2315.

Poggi L, Zolessi FR, Harris WA (2005a) Time-lapse analysis of retinal differentiation. Curr Opin Cell Biol 17:676-681.

Poggi L, Vitorino M, Masai I, Harris WA (2005b) Influences on neural lineage and mode of division in the zebrafish retina in vivo. J Cell Biol 171:991-999.

Reig G, Cabrejos ME, Concha ML (2007) Functions of BarH transcription factors during embryonic development. Dev Biol 302:367-375.

Robu ME, Larson JD, Nasevicius A, Beiraghi S, Brenner C, Farber SA, Ekker SC (2007) p53 activation by knockdown technologies. PLoS Genet 3:e78.

Schuhmacher LN, Albadri S, Ramialison M, Poggi L (2011) Evolutionary relationships and diversification of barhl genes within retinal cell lineages. BMC Evol Biol 11:340.

Skowronska-Krawczyk D, Matter-Sadzinski L, Ballivet M, Matter JM (2005) The basic domain of ATH5 mediates neuron-specific promoter activity during retina development. Mol Cell Biol 25:10029-10039.

Thisse C, Thisse B (2008) High-resolution in situ hybridization to wholemount zebrafish embryos. Nat Protoc 3:59-69.

Vetter ML, Brown NL (2001) The role of basic helix-loop-helix genes in vertebrate retinogenesis. Semin Cell Dev Biol 12:491-498.

Vitorino M, Jusuf PR, Maurus D, Kimura Y, Higashijima S, Harris WA (2009) Vsx2 in the zebrafish retina: restricted lineages through derepression. Neural Dev 4:14.

Wang JC, Harris WA (2005) The role of combinational coding by homeodomain and bHLH transcription factors in retinal cell fate specification. Dev Biol 285:101-115.

Wang SW, Kim BS, Ding K, Wang H, Sun D, Johnson RL, Klein WH, Gan L (2001) Requirement for math5 in the development of retinal ganglion cells. Genes Dev 15:24-29.

Yazulla S, Studholme KM (2001) Neurochemical anatomy of the zebrafish retina as determined by immunocytochemistry. J Neurocytol 30:551-592.

Yeo JY, Lee ES, Jeon CJ (2009) Parvalbumin-immunoreactive neurons in the inner nuclear layer of zebrafish retina. Exp Eye Res 88:553-560.

Zolessi FR, Poggi L, Wilkinson CJ, Chien CB, Harris WA (2006) Polarization and orientation of retinal ganglion cells in vivo. Neural Dev 1:2. 